\title{
Development of a framework for the design of Autonomous Vehicle Storage and Retrieval Systems
}

\begin{abstract}
In today's competitive environment with increasingly faster deliveries and smaller order sizes, material handling providers are progressively developing new solutions. A more recent development in automated material-handling technology for unit load storage and retrieval is the autonomous vehicle storage and retrieval system (AVS/RS). The paper investigates the main design trade-offs for this new solution using simulation, and proposes a comprehensive design framework. Using data from a recently implemented AVS/RS, the application of the proposed framework is presented and the key design differences between the two types of AVS/RS configuration (i.e. "tier captive" versus "tier to tier") are identified.
\end{abstract}

Keywords: warehousing; autonomous vehicle storage and retrieval systems; simulation; automation; design

\section{Introduction}

The autonomous vehicle storage and retrieval system (AVS/RS) is a new automated material-handling technology for unit load storage and retrieval. In a traditional automated storage and retrieval system (AS/RS), unit loads are handled using aislecaptive storage cranes that move simultaneously vertically and horizontally. In an AVS/RS, unit loads are handled by vehicles that move horizontally along rails within the storage racks, while vertical movement is provided by lifts mounted along the rack periphery (Ekren and Heragu 2012).

Based on the format of vehicle assignment to storage tiers, there are two main configurations (Heragu et al. 2011):

- AVS/RS with a "tier to tier" configuration;

- AVS/RS with a "tier captive" configuration.

In the "tier to tier" configuration, vehicles may move from one tier of the storage racks to another using lifts. In the "tier captive" configuration, each vehicle is dedicated to a single tier and therefore cannot move to another one. Lifts are used only to move the unit loads to the destination tiers. 
Evaluating AVS/RS performance is a complex process for warehouse designers due to the compound effect of the kinematic behaviour of vehicles and lift. This complexity may, in turn, be viewed as a function of the rack configuration (i.e. number and length of storage aisles and number of storage tiers), which makes it difficult to design these systems and evaluate their suitability. This complexity is important at the design conceptualisation stage where designers explore alternative configurations and material handling technologies, and estimate the performance of each, before settling on a final design (Heragu et al. 2011). In this initial warehouse design step it is very important to understand the impact of design variables on overall cost and system throughput. It is crucial that an AVS/RS be designed so that it can efficiently handle demand requirements, avoiding bottlenecks and overcapacity (Ekren 2011).

Based on the literature review, studies related to the design of the AVS/RS rack configuration are few and mainly focus on systems with a "tier to tier" configuration and a palletised unit load as the handling unit. This shortcoming is even more significant in light of the fact that several of the main solution providers have implemented numerous AVS/RSs with a "tier captive" configuration and the tote as the handling unit (Marchet et al. 2011b).

Therefore, the focus of this paper is on AVS/RS with a "tier captive" configuration and the tote as the handling unit. With reference to this type of system, the aim of this paper is twofold: first, to study the main design trade-offs; and second, to develop a comprehensive design framework to assist in the identification of the most suitable solution, i.e. that minimises costs, given the user requirements. In other words, given a warehouse problem, the framework should support the warehouse designer to identify the optimal design layout for an AVS/RS in terms of number of 
storage aisles, columns and tiers. According to other studies on AVS/RS, the benefit of a design framework is in the early technology selection, or "conceptualisation" phase of system development (Fukunari and Malmborg 2008, Dallari et al. 2009). Since the successful implementation of an AVS/RS system is highly dependent on an appropriate design, this is a key stage which needs to be better understood.

The remainder of the paper is organised as follows. The main studies of AVS/RS in the literature are presented in Section 2. The AVS/RS evaluated in this paper, the key performance indicators (KPIs) for AVS/RS and the cost modelling are described in Section 3. In Section 4 the simulation model used to evaluate AVS/RS performance is presented and the results of the analysis under different rack configurations and demand levels are discussed. A design framework is developed in Section 5 based on results presented in Sections 3 and 4. Finally, in Section 6 an application of the proposed framework and some design guidelines are presented. Conclusions and future developments are proposed in Section 7.

\section{Literature review}

Studies on AVS/RS may be classified into two main areas of research:

- AVS/RS performance analysis (i.e. throughput and cycle time);

- AVS/RS design criteria.

With regard to the first research area (i.e. AVS/RS performance analysis), the first study was conducted by Malmborg (2002). With reference to a "tier to tier" configuration, a model was proposed to estimate vehicle utilisation and cycle time as a function of the number of storage columns, tiers, vehicles and lifts. 
Following this study, there were several papers on the analysis of AVS/RS performance. In most of these studies, the focus was on AVS/RS with a "tier to tier" configuration and a palletised unit load, and the performance evaluation was generally carried out using analytical models, which were then validated through simulation. For instance, Kuo et al. (2007) modelled the movement of autonomous devices as an M/G/V queue nested within an M/G/L queue to estimate the waiting times for vehicle and lift service, and Zhang et al. (2009) proposed a model that represents storage and retrieval transactions as customers and vehicle-lift pairs as parallel servers. In some other cases, simulation models were developed to evaluate the impact of rack configuration on performance (Ekren et al. 2010) or to identify which factors affect system cycle time by applying Design Of Experiment (Ekren and Heragu 2010).

The "tier captive" configuration has been studied in only two papers, by Heragu et al. (2011) and Marchet et al. (2011b), who presented analytical models based on an open queuing network approach.

The performance of an AVS/RS can be analysed by examining single command cycles only (Kuo et al. 2007, Roy et al. 2009), or both single and dual command cycles (Malmborg 2003, Fukunari and Malmborg 2009), where either a storage or retrieval transaction is completed in the same cycle. According to Zhang et al. (2009) the achievable benefit in terms of efficiency, which is dependent on an increase in the proportion of dual command cycles, is limited for several reasons: first, a high proportion of dual command cycles is difficult to attain and second, storage and retrieval transactions paired in the same cycle are usually associated with different storage tiers.

Papers that address the second research area (i.e. AVS/RS design criteria) can be divided in two categories: i) papers that examine the design issue by comparing the 
AVS/RS with the AS/RS (Automated Storage and Retrieval System) and ii) papers that address the design issue by analysing the system performance according to different rack configurations. Table 1 shows that the majority of contributions in the second research area examined AVS/RS with a "tier to tier" configuration and a palletised unit load as the handling unit. These studies mainly involved simulations that were carried out using the Arena software. In terms of project parameters, the number of storage positions and demand rate are the two synthetic values that were most often used. The number of lifts and vehicles was also considered in some studies. Finally, the average number of experiments examined was 52.

XXXXXXXXXXXXXXXXXXXXXXXXXXXXXXXXXXXXXXXXXXXXXXX

Take in Table 1

\section{XXXXXXXXXXXXXXXXXXXXXXXXXXXXXXXXXXXXXXXXXXXXXXX}

The first study to compare AVS/RS and AS/RS was performed by Malmborg (2002). The author compared the two technologies by varying the system configuration (i.e. storage rack shape, number of lifts and number of vehicles). Fukunari and Malmborg (2008) expanded on the work by Malmborg (2002) by performing an economic comparison between the AVS/RS and AS/RS solutions. Fifteen problem scenarios were considered, with storage capacities ranging from 10,000 to 30,000 storage positions, and transaction demand levels ranging from 100 requests/hour to 300 requests/hour (assuming the use of the Poisson distribution). For each scenario, a comparison was made between the lowest cost AVS/R and AS/R system configurations where vehicle or crane utilisation was below 90\%. Equipment costs were assessed, namely for vehicles and lifts for AVS/RS and cranes and accumulation conveyors for AS/RS. Based on the optimal solutions identified, the authors provide some highlights on AVS/RS rack design with "tier to tier" 
configuration and a palletised unit load as the handling unit: while optimal AS/RS configurations tend to use few but long aisles to minimise the number of cranes, the authors found that optimal AVS/RS configurations tend to use more and shorter aisles to optimise the movement pattern of vehicles and shorter racks to avoid inefficiencies associated with vertical travel. Ekren and Heragu (2011) suggest that better operational performance is achieved with AVS/RS than with AS/RS, under a variety of conditions. Specifically, they found that the AVS/RS configuration that minimises cycle time has a large number of vehicles and aisles (i.e. use of short aisles).

With regard to papers that address the design issue by analysing system performance, Ekren and Heragu (2010) studied the effect of rack configuration (i.e. number of tiers, aisles and columns) on AVS/RS performance in six scenarios by means of simulation. Their analysis confirms that it is better to have many short aisles as opposed to fewer aisles with a larger number of storage columns. Ekren (2011) compared system performance (e.g. average cycle time, average utilisation of lifts and vehicles) and costs (vehicle, lifts and rack costs) for 55 different rack configurations. Depending on the performance required, the optimal configuration was found to vary. According to the authors, the choice of design profile should be based on company/designer priorities (e.g. configuration with average performance measures and low cost or configuration that maximises performance).

Therefore, although there are several studies that address the AVS/RS design issue, a general design framework is lacking. The availability of several tools (Heragu et al. 2011) that allow the designer to evaluate performance for configurations of interest only partially mitigates this need. The choice of which configurations to examine and in what order is, however, left to the warehouse designer. The lack of design frameworks is common both to warehouse design in general and design of 
specific automated solutions (Hassan 2002, Baker and Canessa 2009). In the preliminary phases of the design process for automated solutions such as AS/RS heuristic rules of thumb are often used, which are easy to apply and have an acceptable degree of accuracy (Malborg 2001). An example is Zollinger's rule, which is applicable to the process of identifying a cost-effective storage rack design for a given level of transaction demand (Zollinger 1996).

In summary, several studies have been performed on AVS/RS in the last decade. Those studies chiefly focussed on AVS/RS with a "tier to tier" configuration and a palletised unit load as the handling unit, and assumed that the vehicle moves vertically together with the lift and the number of lifts and vehicles is independent of the number of aisles and the number of tiers on the rack. As the literature review has shown, there is limited information and analysis related to the design criteria for AVS/RS with a "tier captive" configuration and the tote as the handling unit, notwithstanding its more frequent use in a number of industrial tote handling applications. Because of the different ways in which AVS/RS systems function, it is not possible to apply the results from the type of system studied most today (i.e. AVS/RS with a "tier to tier" configuration and the palletised unit load as the handling unit) to the type of system considered in the present study (i.e. AVS/RS with a "tier captive" configuration and the tote as the handling unit). For this reason, a specific study was required.

\section{AVS/RS description}

This section describes the AVS/RS examined, which has a "tier captive" configuration and the tote as the handling unit. The "tier captive" configuration was chosen as it is 
more frequently adopted in warehouses with small handling units that require a high throughput volume.

In the following sub-sections the layout that was assessed, the main notations used in the paper, AVS/RS performance, and the cost modelling process, which was developed based on interviews with material handling providers, are reported.

\subsection{Layout}

Figure 1 shows a representation of an AVS/RS for the handling of totes, while Figure 2 illustrates a single tier. As the figures show, the storage racks are single-deep and double-sided. Each storage position is the same size and can hold one tote. Lifts are mounted at fixed locations at one end of each storage aisle. The input/output (I/O) point is located at the first tier beside each lift. Vehicles move along one dimension only, each within a specific tier of a storage aisle. The number of lifts installed in the system is equal to the number of aisles $(A)$, while the number of vehicles is equal to the product of the number of aisles times the number of tiers $(T)$. The configuration is "tier captive", so the vehicles cannot move from one tier to another. To allow the mutual independence of lift and vehicle, the first position on either side of the storage aisle in all tiers serves as a buffer and is used to manage the transfer of totes between vehicles and lifts (Figure 2). One buffer (called buffer out) handles the totes which have been retrieved, the other one (called buffer in), located on the other side of the storage aisle, handles the totes to be stored. As such, totes (not vehicles) wait for the lift, so lift and vehicle can work independently of one another. It should be noted that the lift is needed in every cycle, even for totes located in storage positions located on the first tier, as the lift permits the transfer of the tote to/from the buffer. 
Take in Figures 1 and 2

XXXXXXXXXXXXXXXXXXXXXXXXXXXXXXXXXXXXXXXXXXXXXXX

As the literature review has shown, performing dual command cycles in an AVS/RS is rather difficult. Therefore, only single command cycles have been considered and, more precisely, this study focuses on single retrieval cycles. This is due to the fact that the retrieval phase is the most critical activity from an organisational viewpoint, as it is directly related to service level and - in contrast to the storage phase - cannot be postponed to a period of low-workload. Furthermore, in some contexts (e.g. AVS/RS used as a dispatching buffer) storage takes place independently of retrieval.

\subsection{Main notations}

The notation used in the remainder of the paper is presented below:

$$
\begin{aligned}
& \lambda_{S}=\text { System retrieval demand rate [retrievals/hour] } \\
& \lambda_{A}=\text { Storage aisle retrieval demand rate [retrievals/hour] } \\
& A=\text { number of storage aisles } \\
& T=\text { number of storage tiers } \\
& C=\text { number of storage columns on each side of a storage aisle } \\
& n=\text { total number of storage positions }\left(2^{*} A^{*} T^{*} C\right) \\
& H_{\text {max }}=\text { maximum height of the racks [m] } \\
& L_{m a x}=\text { maximum length of the racks [m] } \\
& \mu_{w}=\text { unit width of clearance per storage position including allowances [m] } \\
& \mu_{h}=\text { unit height of clearance per storage position including allowances [m] } \\
& v_{V}=\text { average horizontal velocity of vehicle [m/s] } \\
& v_{L}=\text { average vertical velocity of lift [m/s] }
\end{aligned}
$$


$\gamma_{V}=$ vehicle delay due to acceleration, deceleration and braking delay times [s/single command cycle]

$\gamma_{L}=$ lift delay due to acceleration, deceleration and braking delay times [s/single command cycle]

$\varepsilon_{V}=$ time allowance for charging and discharging load from vehicle [s/single command cycle]

$\varepsilon_{L}=$ time allowance for charging and discharging load from lift [s/single command cycle]

\subsection{AVS/RS Key Performance Indicators}

As with other highly-automated systems, the main AVS/RS Key Performance Indicators (KPIs) are flow time and throughput.

Flow time is defined as the total time required by the system to retrieve a tote. It takes into account the time elapsed between the vehicle retrieval request and the moment at which the tote is released by the system, i.e. it reaches the I/O point. As Equation (1) shows, the flow time $(F T)$ is the sum of two variables, namely the tote waiting time for the vehicle $\left(W_{v}\right)$, i.e. the time elapsed between the vehicle retrieval request and the moment at which the vehicle starts to move to the retrieval address, and the cycle time $(\tau)$.

$$
\mathrm{FT}=\mathrm{W}_{\mathrm{v}}+\tau
$$

In turn, $\tau$ is calculated by:

$$
\tau=\tau_{1}+\tau_{2}+\tau_{3}+\tau_{4}+\tau_{5}+\tau_{6}+\tau_{7}+\tau_{8}+\tau_{9}
$$

where: 
$\tau_{1}$ is the time required for the vehicle to travel from the buffer out to the retrieval address;

$\tau_{2}$ is the time required for the vehicle to load the tote at the retrieval address;

$\tau_{3}$ is the time required for the vehicle to move the tote from the retrieval address to the buffer out;

$\tau_{4}$ is the time required for the vehicle to discharge the tote at the buffer out;

$\tau_{5}$ is the tote waiting time for the lift, i.e. the time elapsed from the tote discharging at the buffer out to the moment in which the lift moves from its current position to the retrieval tier;

$\tau_{6}$ is the time required for the lift to travel from the $\mathrm{I} / \mathrm{O}$ point to the retrieval tier;

$\tau_{7}$ is the time required for the lift to load the tote at the buffer out;

$\tau_{8}$ is the time required for the lift to move the tote from the retrieval tier to the $\mathrm{I} / \mathrm{O}$ point;

$\tau_{9}$ is the time required for the lift to discharge the tote at the I/O point.

Throughput represents the number of totes that the system can retrieve per time unit. In conventional automated systems (i.e. AS/RS), throughput may be estimated as the inverse of the average cycle time (Tompkins et al. 2010). In "tier captive" AVS/RS this approach is not applicable due to the use of different resources (i.e. lifts and vehicles). Furthermore, throughput is a function of bottlenecks (i.e. of vehicles or lift). The creation of bottlenecks is a function of the rack configuration and kinematic features of vehicles and lifts.

\subsection{Modelling cost structure}

We propose a cost modelling procedure for an AVS/RS based on the sum of three cost items: equipment cost, rack cost and cost of the space. Uniquely, compared to 
previous studies, rack and space costs were included in this study, resulting in a more comprehensive economic evaluation. This may be useful both for the comparison with other types of automated tote S/R systems (e.g. mini-load) and in studying the most suitable rack configuration. Indeed, AVS/RS racks need rails to allow vehicle movement. As such, AVS/RS racks usually have a significant impact on the overall investment required (according to material handling providers, the rack cost is usually more than $25 \%$ of the overall investment). Moreover, the space required varies as a function of the rack configuration. The greater the vertical extent of the AVS/RS, the less space is required, and thus the cost is reduced.

Costs are expressed in terms of annualised costs. In particular, $A C$ is calculated as follows:

$$
A C=\left(A C_{L}+A C_{V} * T\right) * A+A C_{R} *(2 * T * A * C)+C_{S} * S
$$

where:

$$
\begin{aligned}
& A C_{L}=\text { annualised cost of a lift }[€ / \text { year }] \\
& A C_{V} \quad=\quad \text { annualised cost of a vehicle [€/year] } \\
& A C_{R}=\text { annualised cost of a storage rack position [€/storage } \\
& \text { position*year] } \\
& S \quad=\quad \text { space required }\left[\mathrm{m}^{2}\right] \\
& C_{S}=\quad \text { cost of the space }\left[€ / \mathrm{m}^{2 *} \text { year }\right]
\end{aligned}
$$

The lift cost increases proportionally with the number of aisles, whereas the vehicle cost depends on the combination of the number of aisles $(A)$ and tiers $(T)$. According to Equation (3), if the storage capacity is constant, the number of storage aisles $A$ is the most significant variable. To reduce $A C$, it is necessary to first minimise the number of aisles and then maximise the aisle length to reduce the number of vehicles. 
Finally, the cost of energy consumption could also be included in Equation (3). The expected AVS/RS energy consumption is lower than in AS/RS, as horizontal movements are performed by vehicles, which are lighter than cranes. However, data provided by material handling providers yields only a rough estimate of the energy consumption per cycle, and it is therefore not possible to differentiate energy consumption costs as a function of rack configuration.

\section{Evaluation of AVS/RS performances}

The aim of this section is to study AVS/RS performance (e.g. throughput, flow time and cycle time) by varying the rack configuration.

\subsection{Simulation modelling}

To evaluate the AVS/RS performance, a simulation model was developed using Arena (version 13.0). Figure 3 illustrates the simulation flow chart for a retrieval cycle. A retrieval cycle requires the vehicle in the storage tier where the tote to be retrieved is located. The vehicle first retrieves the tote and then discharges it at buffer out. The lift moves the tote to the output point. During this cycle, it may happen that, for a pending retrieval, the vehicle is already busy or the vehicle has to wait for the buffer out to be empty before discharging the tote (i.e. limited buffer capacity), or the tote has to wait for the lift.

XXXXXXXXXXXXXXXXXXXXXXXXXXXXXXXXXXXXXXXXXXXXXXX

Take in Figure 3

XXXXXXXXXXXXXXXXXXXXXXXXXXXXXXXXXXXXXXXXXXXXXXX

The main assumptions of the simulation model are as follows: 
- Distribution followed by the retrieval demand to the system: Poisson with parameter $\lambda_{s}$. Therefore the time between two subsequent arrivals follows an exponential distribution with parameter $1 / \lambda_{S}$, in accordance with previous studies in the literature, i.e. Fukunari and Malmborg (2008) and Ekren et al. (2010);

- Storage policy: random. In most unit load SR systems, a space-conserving random storage policy is used because of capital cost considerations (Heragu 2008). According to this policy, the probability that a retrieval is required in a certain storage aisle is identical for each aisle and is equal to $1 / A$; similarly, the probability that a retrieval is required in a certain tier is identical for each of them and is equal to $1 / T$. Therefore, the system demand rate $\lambda_{S}$ is evenly distributed among the storage aisles $(A)$ and the storage tiers $(T)$ and the demand rate at each storage aisle follows a Poisson distribution with parameter $\lambda_{A}$ equal to $\lambda_{S} / A$;

- Modelling of lift and vehicle service time: the service times are composed of a variable part and a fixed part. In accordance with the previous literature (Malmborg 2003, Fukunary and Malmborg 2009), the first one (i.e. variable part) is calculated using an average velocity ( $v_{V}$ for the vehicle and $v_{L}$ for the lift), and the second one (i.e. fixed part) is the time for charging and discharging the tote from lift and vehicle, assumed to be constant ( $\varepsilon_{V}$ for the vehicle and $\varepsilon_{L}$ for the lift). In this study, the extra delay due to acceleration, deceleration and braking times is also included in the fixed component $\left(\gamma_{V}\right.$ for the vehicle and $\gamma_{L}$ for the lift), in accordance with other simulation models used to study AVS/RS performance (Ekren at al. 2010);

- Number of totes handled per cycle by lifts and vehicles: 1; 
- Vehicle dwell point policy: point-of-service-completion (POSC);

- Lift dwell point policy: point-of-service-completion (POSC);

- Lift and vehicle dispatching policy: first-come-first serve (FCFS);

- Maximum number of totes in queue at buffer out: 1 .

The simulation model is assumed to be a non-terminating system, making it possible to conduct a steady state analysis (Ekren and Heragu 2010) and, similarly to Fukunary and Malmborg (2008), the specified length of each simulation is 48 hours. The model was run for 20 independent replications. The warm up period is calculated following the procedure proposed by Welch (Law and Kelton 2000), and observations belonging to the warm up period, (3 hours' length on average) have been omitted from the analysis.

The performance assessment was based on the KPIs highlighted in Section 3.3:

- Average throughput;

- Average flow time, considering both the overall performance and its two components (i.e. tote average waiting time for the vehicle at the retrieval address, and average cycle time), and identifying the contribution of the average waiting time for the lift at the buffer out.

\subsection{Main simulation data}

Similarly to previous studies (e.g. Kuo et al. 2007), the analysis was performed by considering different combinations of storage capacity and retrieval demand level. A storage capacity value $n$ equal to 10,000 storage positions and five retrieval demand rates at each storage aisle $\lambda_{A}$ (i.e. 100, 250, 500, 750, 1000 retrievals/hour) were 
considered, in order to evaluate performance based on different resource utilisation levels.

In the AVS/RS design there are usually some physical constraints (Ekren and Heragu 2010). Specifically, in AVS/RS for the handling of totes the maximum height corresponds to an industrial building height. Therefore, a maximum system height of $10 \mathrm{~m}$ was assumed. The maximum length assigned to the storage aisles was $80 \mathrm{~m}$, which corresponds to the maximum length for AVS/RSs currently in place.

Nine rack configurations were analysed, as a combination of three values of $T$ (i.e. 8,10 and 12) and three values of $A$ (i.e. 4,8 and 16). Here, the level of the $C$ variable varies according to $T$ and $A$ values, and $\lambda_{S}$ is equal to $\lambda_{A} * A$. Table 2 reports the three rack dimensions, the expected throughput of the vehicles in one aisle $\left(E T H_{V}\right)$ and the expected throughput of the lift $\left(E T H_{L}\right) . E T H_{V}$ and $E T H_{L}$ may be computed as follows:

$$
\begin{aligned}
& E T H_{V}=\frac{3600}{\tau_{V}} * T \\
& E T H_{L}=\frac{3600}{\tau_{L}}
\end{aligned}
$$

According to the random storage policy hypothesis, $\tau_{V}$ is equal to the length of a retrieval cycle performed by a vehicle at the midpoint of the aisle:

$$
\tau_{V}=\frac{\mu_{w} * C}{2 * v_{V}} * 2+\gamma_{V}+\varepsilon_{V}
$$

Similarly, $\tau_{L}$ is equal to the length of a retrieval cycle performed by a lift at the midpoint of its maximum distance travelled:

$$
\tau_{L}=\frac{\mu_{h} *(T-1)}{2 * v_{L}} * 2+\gamma_{L}+\varepsilon_{L}
$$


Table 2 also reports the type of resource (i.e. lift or vehicles) that is expected to be the bottleneck for each rack configuration, based on the $E T H_{V}$ and $E T H_{L}$ results. The expected throughput of each storage aisle $\left(E T H_{A}\right)$ and the expected throughput of the system $\left(E T H_{S}\right)$ are defined as:

$$
\begin{gathered}
E T H_{A}=\min \left(E T H_{V}, E T H_{L}\right) \\
E T H_{S}=A * E T H_{A}
\end{gathered}
$$

The combination of the five values of retrieval demand rate at each storage aisle $\left(\lambda_{A}\right)$ and the nine rack configurations lead to the analysis of 45 types of experiments. Additional data used in all simulation runs are reported in Table 3. XXXXXXXXXXXXXXXXXXXXXXXXXXXXXXXXXXXXXXXXXXXXXXX

Take in Tables 2 and 3

XXXXXXXXXXXXXXXXXXXXXXXXXXXXXXXXXXXXXXXXXXXXXXX

\subsection{Simulation results}

Simulation results are reported in Table 4. To enhance readability, they refer to a single aisle. Under the assumption of random storage policy, it should be noted that the behaviour of each aisle is identical.

XXXXXXXXXXXXXXXXXXXXXXXXXXXXXXXXXXXXXXXXXXXXXXX

Take in Table 4

\section{XXXXXXXXXXXXXXXXXXXXXXXXXXXXXXXXXXXXXXXXXXXXXXX}

The following observations on the functioning of the AVS/RS system can be made based on these results: 
- The throughput of one aisle may be estimated analytically by identifying the system bottleneck (i.e. lift or vehicles), according to Equation (8). In fact, looking at Table 2 and Table 4, the simulated throughput performance corresponds to the expected throughput of the vehicle in one aisle $\left(E T H_{V}\right)$ where $E T H_{V}$ is lower than $E T H_{L}$ (e.g. experiments 28, 38 and 40). Conversely, the simulated throughput performance corresponds to the expected throughput of the lift $E T H_{L}$ where $E T H_{L}$ is lower than $E T H_{V}$ (e.g. experiments 39, 41 and 42). The average cycle time and average flow time are hardly predictable $a$ priori. These may be obtained by using simulation or analytical modelling (i.e. modelling the creation of queues in the system);

- The throughput performance corresponds to $\lambda_{A}$ where the expected throughput $E T H_{A}$ is higher than the demand rate $\lambda_{A}$, (e.g. experiments 10-18);

- For experiments $19,28,31$ and $37-45$, it may be observed that where the demand level $\lambda_{A}$ is similar to the expected throughput $E T H_{A}$, the high utilisation of lifts and vehicles leads to the creation of queues and, therefore, to long average cycle times and average flow times. In general, the increase in the average cycle time is related to the waiting time for the lift at buffer out, whereas the increase in average flow time may be correlated to both a long waiting time for the lift at buffer out, and a long waiting time for the vehicle at the retrieval address. For instance, the average waiting time for the vehicle ranges from $1.28 \mathrm{~s}$ to $45.50 \mathrm{~s}$ where there is a low demand rate (e.g. experiments $10-18$ ), and from $8.10 \mathrm{~s}$ to $17,534 \mathrm{~s}$ where there is a high demand rate (e.g. experiments 37-45). For those cases with vehicle or lift utilisation level below 0.9 the related waiting times are reasonably low. For instance, in the experiment 22 the average flow time is $133.27 \mathrm{~s}$, which is the sum of the 
average waiting time for the vehicle (i.e. $74.61 \mathrm{~s}$ ) and the average cycle time (i.e. $58.66 \mathrm{~s}$, including the average waiting time for the lift of $2.26 \mathrm{~s}$ ). These results confirm previous studies that use a resource utilisation level of 0.9 during the design phase of AVS/RS systems (Fukunary and Malmborg 2008, Kuo et al. 2007);

- The rack configuration, and specifically the relationship between the number of tiers $(T)$ and number of storage columns $(C)$, impacts on the creation of the bottleneck. In the presence of high racks the bottleneck is usually caused by the lift, which has to travel longer vertical distances and serves a greater number of tiers (e.g. please refer to the throughput performance in experiments 43,44 and 45). When the rack height decreases, the storage aisle tends to be longer, and the bottleneck may be caused by the vehicles, as they have to travel longer distances (e.g. please refer to the throughput performance in experiments 37, 38 and 40). However, as shown in Table 2, this does not occur when $A=16$. In this case, even with the number of tiers equal to 8 , the bottleneck is still caused by the lift: indeed, in this case, the aisle length $(C=40)$ allows vehicles to quickly move the totes to the buffer out;

- The maximum throughput of a single aisle does not necessarily correspond to the configuration with the maximum possible rack height. Such a result is shown in Figure 4, which illustrates the expected throughput per aisle $\left(E T H_{A}\right)$, varying $T$ and $A$. It can be noted that, when the number of aisles is 4 , the throughput performance of a single aisle tends to increase as $T$ increases. In fact, as shown in Table 2, for a number of tiers ranging from $T=8$ (i.e. rack configuration 1) to $T=10$ (i.e. rack configuration 4 ), $E T H_{V}$ increases as a result of shorter travel distances, although the bottleneck is still caused by the 
vehicle. Moving from $T=10$ to $T=12$ (i.e. rack configuration 7), a further reduction in $C$ leads to a change in the creation of the bottleneck, which is now caused by the lift. A different result is obtained when $A=16$ : the throughput performance of the single aisle tends to decrease as the number of levels increases. This is due to the fact that the lift causes the bottleneck (i.e. rack configuration 3, 6 and 9), and therefore the increase in rack height worsens lift performance.

\section{XXXXXXXXXXXXXXXXXXXXXXXXXXXXXXXXXXXXXXXXXXXXX}

Take in Figure 4

XXXXXXXXXXXXXXXXXXXXXXXXXXXXXXXXXXXXXXXXXXXXX

\section{A framework for the AVS/RS design}

As previously illustrated, the rack configuration affects both cost structure and system performance. In Figure 5 an AVS/RS design framework is presented, the purpose of which is to facilitate the identification of the suitable rack configuration, i.e. the rack configuration that meets user requirements (i.e. storage capacity and throughput capacity) at a minimum cost, given the required service level (i.e. average flow time) and the physical constraints. The framework aims to facilitate the task of designers, by outlining important design guidelines and helping designers to make informed decisions. The framework is derived from a literature review on warehouse design conceptualisation, the analysis of system performance (Section 4) and the modelling of the cost structure (Section 3.4).

XXXXXXXXXXXXXXXXXXXXXXXXXXXXXXXXXXXXXXXXXXXXXXX

Take in Figure 5

XXXXXXXXXXXXXXXXXXXXXXXXXXXXXXXXXXXXXXXXXXXXXXX 
In step 1 the size of the storage position is defined as a function of the product features (e.g. in the pharmaceutical industry product totes are smaller in size than in the food industry), and the technology used by the vehicle to charge and discharge the tote.

Once the size of the storage position has been defined, an initial solution (i.e. system configuration) is identified (step 2). As the purpose of this design framework is cost minimisation, this solution corresponds to the rack configuration which presents the minimum cost among those meeting the storage capacity requirements. Beginning with the maximum number of levels $T_{\max }$ and storage columns $C_{\max }$, the minimum number of aisles $A_{\min }$ that satisfy the physical constraints and the required storage capacity is calculated in step 2:

$$
\begin{gathered}
T_{\max }=\text { lower integer part }\left[H_{\max } / \mu_{h}\right] \\
C_{\max }=\text { lower integer part }\left[L_{\max } / \mu_{w}\right] \\
A_{\min }=\text { upper integer part }\left[n /\left(2 * T_{\max } * C_{\max }\right)\right]
\end{gathered}
$$

The initial rack configuration determined by $T_{\max }, C_{\max }$ and $A_{\min }$ might give rise to an overcapacity in terms of the number of storage positions and vehicle fleet size. Therefore, the rack configuration is determined in step 2 as follows:

$$
\begin{gathered}
\qquad A_{0}=A_{\text {min }} \\
T_{0}=\text { upper integer part }\left[n /\left(2 * A_{\min } * C_{\max }\right)\right] \\
C_{0}=\text { upper integer part }\left[n /\left(2 * A_{\min } * T_{0}\right)\right]
\end{gathered}
$$

In step 3, the expected system throughput $E T H_{S}$ is calculated in order to verify whether the current solution satisfies the constraint on the throughput target. Based on 
the results of Section 4.3, the expected system throughput may be estimated according to the Equation (9). In general, during the system design phase there is usually the need to set a constraint on the resource utilisation level (i.e. lower than 1). Therefore, the throughput requirement should be appropriately increased, to obtain a resource utilisation level that is reasonable. Based on the results of Section 4.3, a throughput target that is the ratio between the throughput requirement and a resource utilisation level of 0.9 is proposed. This will limit the creation of queues and, as a consequence, both cycle time and flow time will be relatively low, thus satisfying the service level requirement.

If the solution meets the throughput target, then the procedure ends with the calculation of the annualised cost of the proposed configuration, and the average flow time by means of simulation (step 4). If the throughput of the proposed rack configuration is lower than the target value, the design procedure is not yet complete. The increase of $E T H_{S}$ is obtained as a function of the bottleneck position. According to Section 4.3 , if the bottleneck is caused by the lift, then the number of aisles should be increased (i.e. $A=A+1$ ) and $T$ is calculated, given $C_{\max }$ and the current value of $A$ (step 5) by:

$$
T=\text { upper integer part }\left[n /\left(2 * A * C_{\max }\right)\right]
$$

Subsequently, the value of $C$ is calculated, using $T$ calculated above and the current value of $A$ :

$$
C=\text { upper integer part }[n /(2 * A * T)]
$$

Following step 5, the procedure continues with a return to step 3. 
Conversely, if the bottleneck is due to the vehicles, then the procedure moves to step 6. In this latter case, the suggested procedure is to increase $T$, thus increasing $E T H_{V}$. This reduces the aisle length and, at the same time, increases the number of vehicles per aisle. Before performing step $6, T$ is checked to see if it is equal to $T_{\max }$. If $T$ is equal to $T_{\max }$ the procedure is to return to step 5 and then to step 3 , without completing step 6. If not, the number of levels is increased (i.e. $T=T+1$ ) and $C$ is then calculated, using the current values of $A$ and $T$ according to Equation (17). After step 6 , the procedure returns to step 3 .

As suggested in literature, once the most appropriate rack configuration has been identified, fine tuning of the solution through simulation can take place, in order to improve the system performance, for example by considering alternative operating policies.

\section{Framework application}

Using the data from an implementation of an AVS/RS with a "tier captive" configuration in the United Kingdom by Knapp, a material handling provider, we show an application of the proposed design framework. The aim of presenting this application is twofold: to provide an experimental validation of the framework and to derive some guidelines for AVS/RS design.

The warehouse used in this analysis is a distribution centre that serves all the stores in the UK for a retailer in the apparel sector. The warehouse is made up of various storage areas. Operationally, once a customer order has been received, item picking takes place in the storage areas. Picked items are packaged in customer boxes. When they are ready, the customer boxes are stored in the AVS/RS to await shipment. More detailed data about the company have been withheld for confidentiality reasons. 
The framework was first applied to the specific case. Then, several constraints related to the building housing the AVS/RS were removed in order to apply the model to evaluate 9 scenarios. Sixty four rack configurations were assessed for each scenario.

\subsection{Case study}

The user requirements for this case study were 9500 storage positions (i.e. customer boxes) and a system throughput of 4000 retrievals/hour (based on the demand peak, an average system throughput of 3000 retrievals/hour is sufficient). In order to respect shipping windows, the average flow time for a box must not be high (i.e. less than 5 minutes). Based on the product features, the width and the height of each storage position are 0.6 and 0.7 metres, respectively. Due to the storage area's height limitation, the maximum rack height is $11 \mathrm{~m}$ (i.e. $T_{\max }$ is equal to 15 ). Similarly, the maximum rack length is $40 \mathrm{~m}$ (i.e. $C_{\max }$ equal to 66).

As shown in Table 5, sixty-four rack configurations were assessed, as a result of eight different aisle numbers $(A)$ and eight different tier numbers $(T)$. For each configuration, the expected system throughput, the average flow time and the annualised cost were calculated. $E T H_{S}$ values were estimated using Equation (9). The average flow time was obtained through simulation. The cost of each solution was calculated as shown in Section 3.4, assuming 10 years of service and a $10 \%$ interest rate, In accordance with Marchet et al. (2011a). Table 6 reports the unit costs obtained from interviews with material handling providers.

XXXXXXXXXXXXXXXXXXXXXXXXXXXXXXXXXXXXXXXXXXXXXXX

Take in Tables 5 and 6

XXXXXXXXXXXXXXXXXXXXXXXXXXXXXXXXXXXXXXXXXXXXXXX 
The minimum cost configuration that satisfies all the requirements has 6 storage aisles and 12 tiers, with a calculated $A C$ of $237,595 €$. The results of the simulation confirm that the existing system meets the throughput requirement, without adversely affecting the service level. In fact, the average cycle time is $0.81 \mathrm{~min}$, and the average flow time is $1.28 \mathrm{~min}$. As expected, resource utilisation was found to be less than 0.9 , i.e. 0.57 for the vehicles and 0.82 for the lifts. The minimum cost configuration can be obtained by following the proposed design framework. In fact, application of the framework involves an initial configuration (step 2) with $A_{\min }=5, T_{0}$ $=15$ and $C_{0}=64$. This configuration, in which the average cycle time and average flow time are both 1.95 hours, is not acceptable as it does not satisfy the throughput target (i.e. 3678 retrievals/hour compared to a throughput target of 4000/0.9 which is equal to 4445 retrievals/hour). Because the bottleneck in this configuration is caused by the lift, according to the framework, the number of aisles should be increased and a configuration with $A=6, T=12$ e $C=66$ should be evaluated, which results in the minimum cost solution. It should be noted that the minimum cost rack configuration identified by applying the framework corresponds with that implemented in the studied case.

\subsection{Scenario analysis}

Additional analyses were performed by specifying different combinations of maximum rack lengths (i.e. maximum number of storage columns) and throughput requirements. More specifically, three values for the maximum number of storage columns (i.e. 55, 75 and 95) and throughput requirements (i.e. 3000, 4000 and 5000 retrievals/hour) were considered. Therefore, 9 scenarios were evaluated (see Table 7). All the other values remained the same as those used in the case study (see Section 
6.1). The storage capacity value $n$ is equal to 9500 storage positions and maximum number of tiers is 15 . The estimate of performance (i.e. throughput and flow time) was conducted in the same manner as in Section 6.1.

\section{XXXXXXXXXXXXXXXXXXXXXXXXXXXXXXXXXXXXXXXXXXXXXXX}

Take in Table 7

\section{XXXXXXXXXXXXXXXXXXXXXXXXXXXXXXXXXXXXXXXXXXXXXXX}

Table 8 shows the results of applying the framework to scenarios where $C_{\max }$ is 55 (i.e. scenarios 1, 2 and 3). The first solution identified by the framework always produces the minimum cost from among those solutions that satisfy the physical constraints (rack configuration 24 in Table 8). This rack configuration is already the final solution for scenario 1 in that $E T H_{S}$ is greater than $T H_{\text {target }}(3,334$ retrievals/hour). For scenarios 2 and 3 , in which the throughput capacity had to be increased to satisfy the service requirements, the framework suggests to increase the number of aisles since the bottleneck occurs at the lift. This means to evaluate only rack configuration 30 in scenario 2 and rack configurations 30 and 36 in the scenario 3 . In all three scenarios, the optimal solution identified using the framework is the minimum cost solution (among those that respect the layout and service constraints).

In terms of the results for the other six scenarios (Tables 9 and 10) a similar search process was observed. Furthermore, as $C_{\max }$ increases, the framework's starting solution becomes focussed on a smaller number of aisles. This implies that the initial solution has a lower throughput capacity and a greater number of alternatives are considered prior to identifying the optimal solution (from 3 rack configurations assessed in scenario 3 up to 8 assessed in scenario 9).

Generalising the results of these 576 experiments (64 rack configurations $* 9$ scenarios), it can be concluded that the proposed design framework always identifies 
the optimal solution from among the potential solutions based on the physical and service constraints. The framework application involves the computation and assessment for only a small number of alternative rack configurations (e.g. 8 for scenario 9 and only 1 configuration for scenario 1), such that simulation was needed only to further evaluate the performance of identified configuration and to fine-tune it. This derives from the fact that the framework allows the warehouse designer to assess alternative rack configurations in an intelligent manner. It should be noted that the framework is dependent on the assumptions made with respect to cost structure, particularly the relationship between vehicle cost and lift cost. However, any significant change in this respect is, at the moment, considered to be highly improbable by material handling providers.

\section{XXXXXXXXXXXXXXXXXXXXXXXXXXXXXXXXXXXXXXXXXXXXXXX}

Take in Tables 8, 9 and 10

\section{XXXXXXXXXXXXXXXXXXXXXXXXXXXXXXXXXXXXXXXXXXXXXXX}

Based on the analysis of the scenarios presented above, the following design guidelines were identified. First, the most appropriate configuration differs from that for AVS/RS with a "tier to tier" configuration, which has been presented in the literature. While it is better to have a larger number of short aisles in systems with a "tier to tier" configuration, this study shows that in systems with a "tier captive" configuration a smaller number of longer aisles is more appropriate.

However, for both configurations (i.e. "tier to tier" and "tier captive"), the optimal solution may not be one that maximises rack height, making full use of the height of an industrial building. 


\section{Conclusions and further research}

This study focused on the analysis of AVS/RS performance for product totes with a "tier captive" configuration. Performance assessment is highly complex due to the compound effect of the kinematic behaviour of the vehicles and the lift. In turn, this complexity is a function of the rack configuration (i.e. number and length of storage aisles and number of tiers).

First, examination of the AVS/SR cost structure showed that cost minimisation may be achieved by first minimising the number of aisles, given the physical building constraints, and then maximising aisle length to reduce the number of vehicles required.

Second, the effect of rack configuration on AVS/RS performance was studied through simulation using the Arena software. Simulation results confirm that throughput may be estimated analytically by identifying the system bottleneck (i.e. lift or vehicles). Analytical formulas for estimating throughput were then defined. However the average cycle time and average flow time are more hardly predictable a priori. Simulation results show that when the resource utilisation level (i.e. lifts or vehicles) is below 0.9 the impact of waiting times on flow time is minimal, and therefore flow time is not much greater than cycle time. When resource utilisation exceeds 0.9 , system performance decreases appreciably. Therefore, in the design phase, a target value of 0.9 for resource utilisation should be used.

Finally, the simulation results illustrate the effect of rack configuration on throughput performance. The lift tends to be the cause of the bottleneck where the configuration has high racks. Conversely, for a given storage capacity, vehicles tend to create the bottleneck as the number of storage tiers is reduced. As a consequence, the maximum throughput for an aisle does not necessarily correspond to the configuration 
with the maximum rack height. Furthermore, the results confirm the relationship between system throughput and the two main design variables, i.e. number of aisles (A) and number of tiers $(T)$ : to increase the system throughput, the number of tiers $(T)$ needs to be adjusted when vehicles create the bottleneck; conversely the number of aisles (A) should be adjusted if the bottleneck is caused by the lift. Recognising this pattern makes it possible to increase the system throughput performance while avoiding overcapacity (i.e. at a minimal incremental cost).

The in-depth examination of system performance with varying rack configurations, together with the cost structure modelling, were the basis for the development of a design framework for AVS/RS with a "tier captive" configuration and the tote as the handling unit. This framework is a useful tool for warehouse designers, as it permits a rapid identification of the most appropriate rack configuration. The primary purpose of the framework is to assist designers in the conceptualisation phase of system development. It makes it possible to design AVS/RS that satisfy user requirements while avoiding overcapacity and then decreasing the overall system cost.

Finally, an application of the framework was presented which used data from an AVS/RS in the United Kingdom. The results of over 576 experiments show that the proposed design framework always identifies the optimal solution from among the potential solutions, given the physical and service constraints, and involves the analysis and assessment of a small number of alternative rack configurations. Finally, the results have highlighted the main differences in design approach between the "tier to tier" configuration studied more frequently in the literature and the "tier captive" configuration examined in this paper. In "tier to tier" systems, where vehicles may access locations on different storage tiers and a lift is not dedicated to a single aisle, it 
is more appropriate to have a high number of short aisles. However, the optimal configuration for a "tier captive" AVS/RS (i.e. one lift for each aisle and as many vehicles as the number of tiers) tends to have a smaller number of longer aisles.

This first step in the research involved an assessment of the performance of AVS/RS under various design scenarios. Using the proposed design framework, it will be possible, through future research, to perform an economic comparison of AVS/RS with other automated systems, such as miniload. In addition, it will be possible to compare the results with empirical results obtained from an analysis of the industrial applications of these automated solutions and, if needed, extend the analysis to other aspects of warehousing such as picking activity. 


\section{References}

Baker, P. and Canessa, M., 2009. Warehouse design: a structured approach. European Journal of Operational Research, 193 (2), 425-436.

Dallari, F., Marchet, G., and Melacini M., 2009. Design of order picking system. International Journal of Advance Manufacturing Technology, 42 (1), 1-12.

Ekren, B.Y., 2011. Performance evaluation of AVS/RS under various design scenarious: a case study. International Journal of Advance Manufacturing Technology, 55 (9-12), 1253-1261.

Ekren, B.Y. and Heragu, S.S., 2010. Simulation based regression analysis for rack configuration of autonomous vehicle storage and retrieval systems. International Journal of Production Research, 48 (21), 6257-6274.

Ekren, B.Y. and Heragu, S.S., 2011. Performance comparison of two material handling systems: AVS/RS and CBAS/RS. International Journal of Production Research, DOI:10.1080/00207543.2011.588627.

Ekren, B.Y. and Heragu, S.S., 2012. A new technology for unit-load automated storage system: autonomous vehicle storage and retrieval system. In: R. Manzini, Warehousing in the Global Supply Chain. London: Springer, 285-340.

Ekren, B.Y., Heragu, S.S., Krishnamurthy, A., and Malmborg, C.J., 2010. Simulation based experimental design to identify factors affecting performance of AVS/RS. Computers \& Industrial Engineering, 58 (1), 175-185.

Fukunari, M. and Malmborg, C.J., 2008. An efficient cycle time model for autonomous vehicle storage and retrieval systems. International Journal of Production Research, 46 (12), 3167-3184.

Fukunari, M. and Malmborg, C.J., 2009. A network approach for evaluation of performance measures in autonomous vehicle storage and retrieval systems. European Journal of Operational Research, 193 (1), 152-167.

Hassan, M., 2002. A framework for the design of warehouse layout. Facilities, 20 (13/14), 432-440.

Heragu, S.S., 2008. Facilities design. Clermont: CRC Press.

Heragu, S.S., et al., 2011. Analytical models for analysis of automated warehouse material handling systems. International Journal of Production Research, 49 (22), 6833-6861.

Kuo, P.H., Krishnamurthy, A., and Malmborg, C.J., 2007. Design models for unit load storage and retrieval systems using autonomous vehicle technology and resource conserving storage and dwell point policies. Applied Mathematical Modelling, 31 (10), 2332-2346.

Kuo, P. H., Krishnamurthy, A., and Malmborg, C.J. 2008. Performance modelling of autonomous vehicle storage and retrieval systems using class-based storage policies. International Journal of Computer Applications in Technology, 31 (3/4), 238-248.

Law, A.M. and Kelton, W.D., 2000. Simulation Modeling and Analysis. 3rd ed. New York: McGraw-Hill.

Malmborg, C.J., 2001. Rule of thumb heuristics for configuring storage racks in automated storage and retrieval system design. Int. J. Production Research, 39 (3), 511-527.

Malmborg, C.J., 2002. Conceptualizing tools for autonomous vehicle storage and retrieval systems. International Journal of Production Research, 40 (8), 18071822. 
Malmborg, C.J., 2003. Interleaving dynamics in autonomous vehicle storage and retrieval systems. International Journal of Production Research, 41 (5), 10571069.

Marchet, G., Melacini, M., and Perotti, S., 2011a. A model for design and performance estimation of pick-and-sort order picking systems. Journal of Manufacturing Technology Management, 22 (2), 261-282.

Marchet, G., et al., 2011b. Analytical model to estimate performances of autonomous vehicle storage and retrieval systems for product totes. International Journal of Production Research, DOI: 10.1080/00207543.2011.639815.

Roy, D., et al., 2009. Impact of Zones on Throughput and Cycle Times in Warehouses with Autonomous Vehicles. Proceedings of the 5th ed. Annual IEEE Conference on Automation Science and Engineering, Bangalore, India, August 22-25, 449454.

Tompkins, J.A., et al., 2010. Facilities Planning. New York: John Wiley and Sons.

Zhang, L., et al., 2009. Variance-based approximations of transaction waiting times in autonomous vehicle storage and retrieval systems. European Journal of Industrial Engineering, 3 (2), 146-169.

Zollinger, H.A., 1996. Expanded methodology to concept horizontal transportation problem solutions. In.: Graves, R.J. et al., Progress in Material Handling Research. Material Handling Institute, Charlotte, North Carolina, 651-663. 


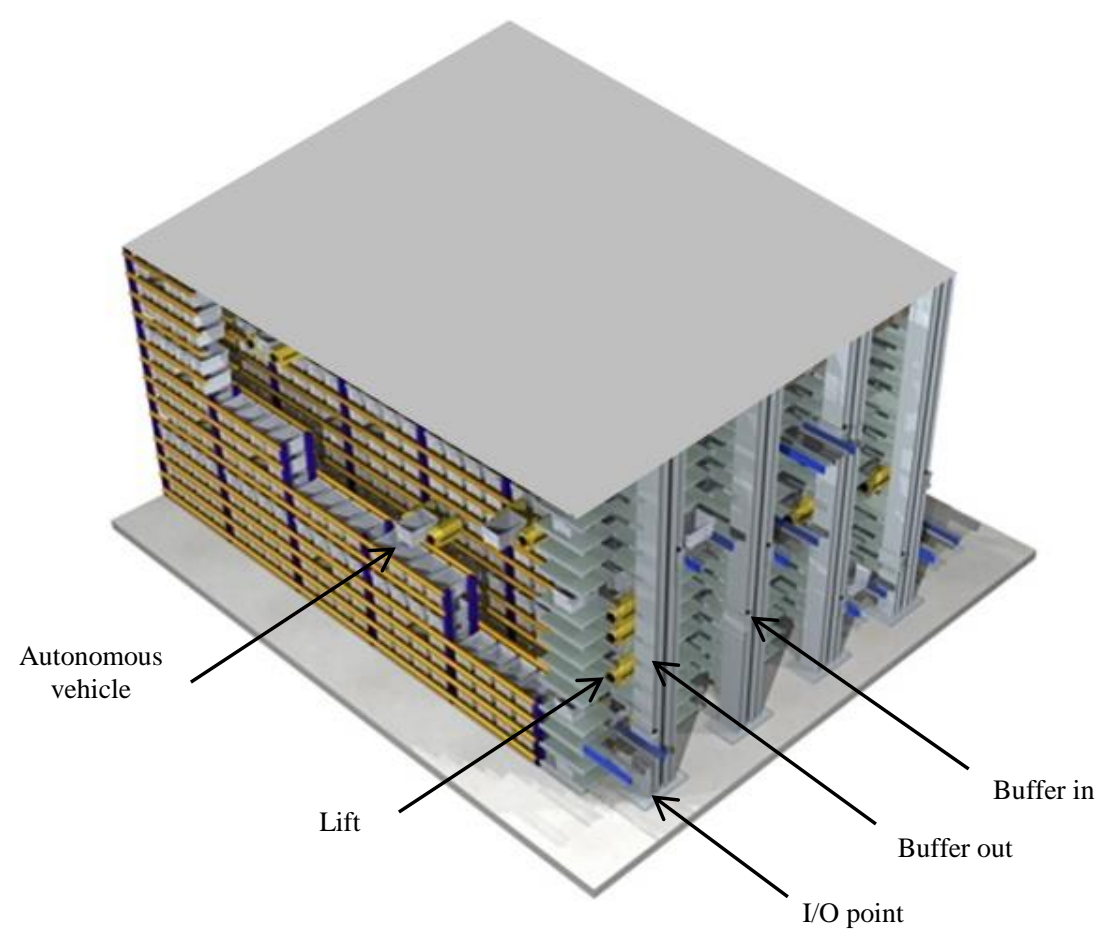

Figure 1. AVS/RS with a "tier captive" configuration and the tote as the handling unit.

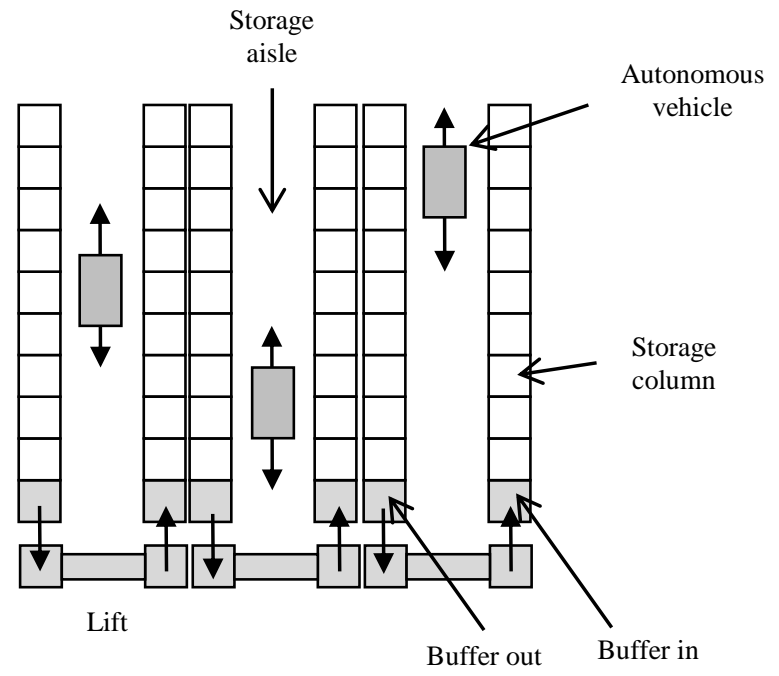

Figure 2. Depiction of a tier of an AVS/RS with a "tier captive" configuration and the tote as the handling unit. 


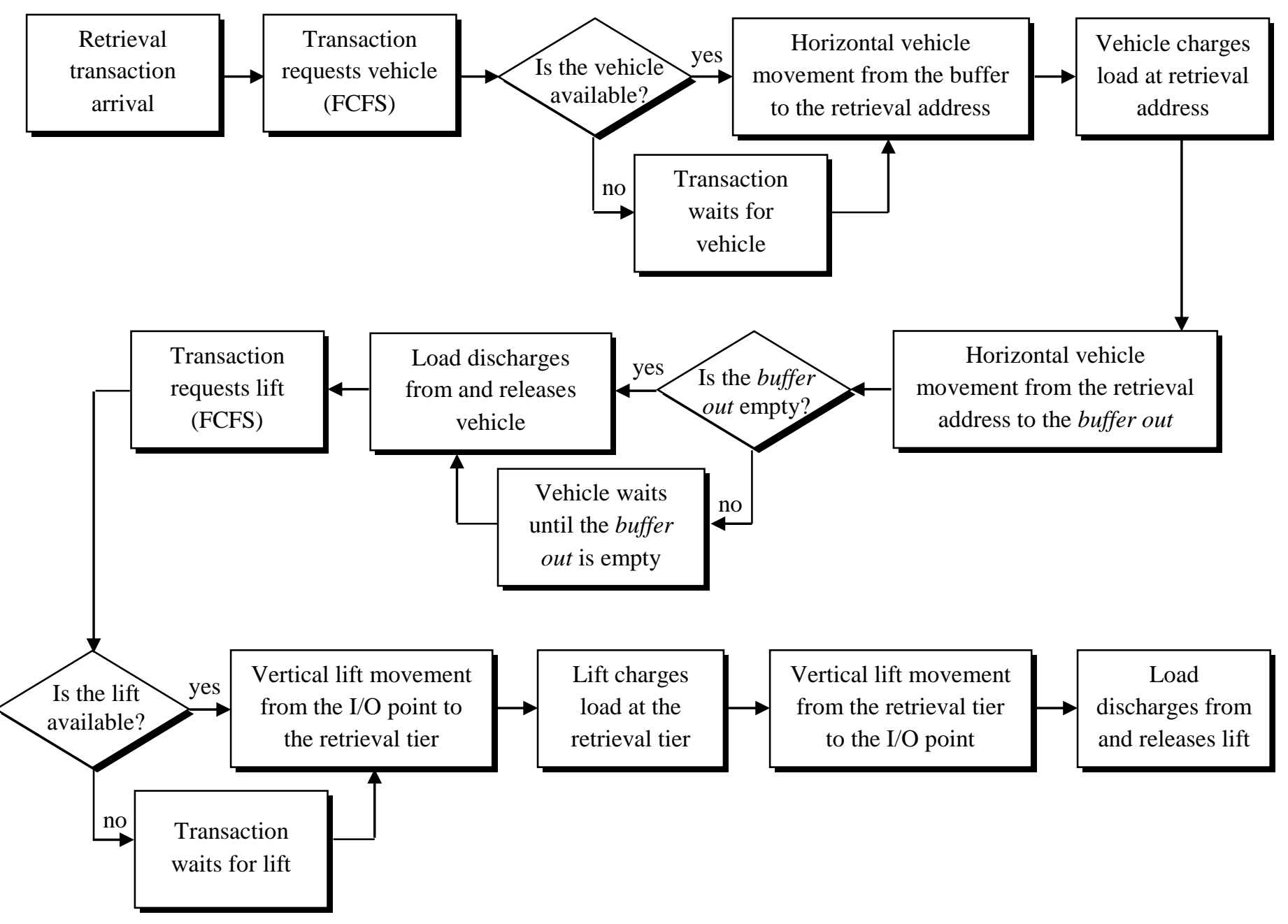

Figure 3. Simulation flow chart for retrieval cycle.

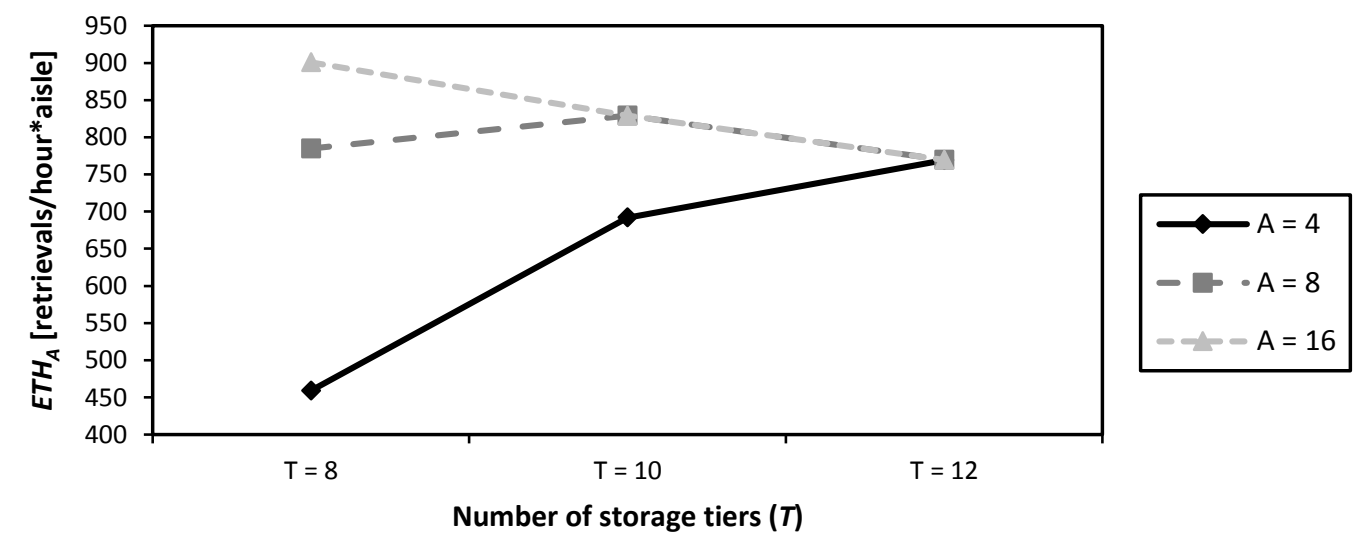

Figure 4. Expected throughput per aisle $\left(E T H_{A}\right)$ varying the number of storage tiers $(T)$ and number of aisles $(A)$, assuming a number of storage locations per aisle equal to 2500 when $A=4,1250$ when $A=8$, and 625 when $A=16$. 


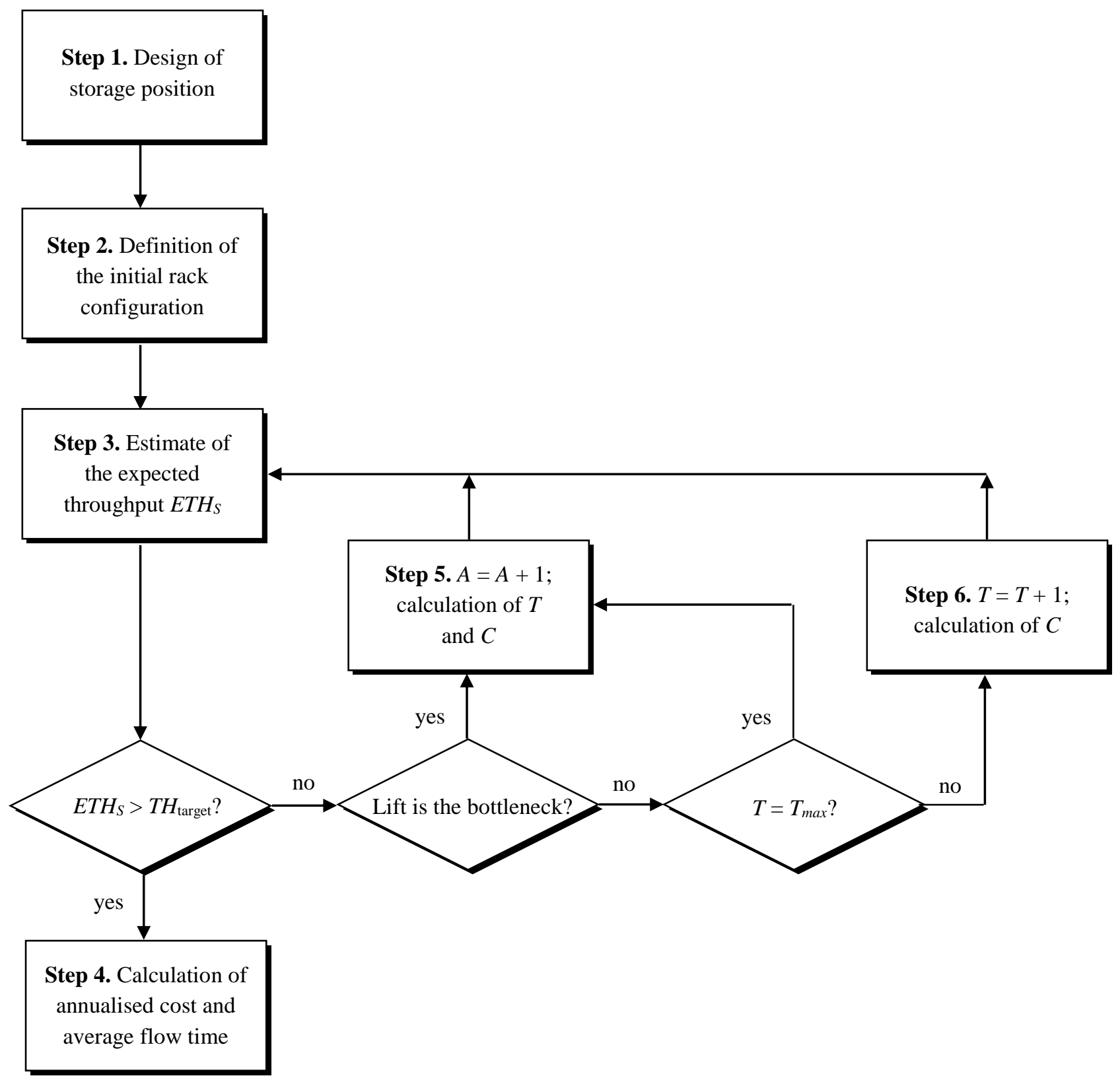

Figure 5. Design framework for the design of an AVS/RS with a "tier captive" configuration. 
Table 1. Overview of the main studies on AVS/RS design criteria.

\begin{tabular}{|c|c|c|c|c|c|c|}
\hline Paper & $\begin{array}{l}\text { Category } \\
\text { of the } \\
\text { paper }\end{array}$ & Configuration & Methodology & Project parameters & $\begin{array}{l}\text { Number of } \\
\text { experiments }\end{array}$ & Unit load \\
\hline $\begin{array}{l}\text { Malmborg } \\
\text { (2002) }\end{array}$ & A & "tier to tier" & $\begin{array}{c}\text { Analytical } \\
\text { model validated } \\
\text { through } \\
\text { simulation }\end{array}$ & $\begin{array}{c}\text { Number of storage } \\
\text { positions }(10,000) \text { and } \\
\text { demand rate }(150 \\
\text { transactions/hour })\end{array}$ & 25 & $\begin{array}{c}\text { Palletised } \\
\text { unit load }\end{array}$ \\
\hline $\begin{array}{l}\text { Kuo et al. } \\
\text { (2007) }\end{array}$ & A & "tier to tier" & $\begin{array}{c}\text { Analytical } \\
\text { model validated } \\
\text { through } \\
\text { simulation using } \\
\text { AutoMod } \\
\text { software }\end{array}$ & $\begin{array}{c}5 \text { number of storage } \\
\text { positions }(10,000 \text { to } \\
30,000 \text { with steps of } 5,000) \\
\text { and } 3 \text { demand rates }(100 ; \\
200 ; 300 \\
\text { transactions/hour) }\end{array}$ & 15 & $\begin{array}{c}\text { Palletised } \\
\text { unit load }\end{array}$ \\
\hline $\begin{array}{l}\text { Fukunari } \\
\text { and } \\
\text { Malmborg } \\
\text { (2008) }\end{array}$ & A & "tier to tier" & $\begin{array}{c}\text { Analytical } \\
\text { model validated } \\
\text { through } \\
\text { simulation using } \\
\text { AutoMod } \\
\text { software }\end{array}$ & $\begin{array}{c}5 \text { number of storage } \\
\text { positions }(10,000 \text { to } \\
30,000 \text { with steps of } 5,000) \\
\text { and } 3 \text { demand rates }(100 ; \\
200 ; 300 \\
\text { transactions/hour) }\end{array}$ & 15 & $\begin{array}{c}\text { Palletised } \\
\text { unit load }\end{array}$ \\
\hline $\begin{array}{l}\text { Ekren and } \\
\text { Heragu } \\
(2010)\end{array}$ & B & "tier to tier" & $\begin{array}{l}\text { Simulation } \\
\text { model using } \\
\text { Arena software }\end{array}$ & $\begin{array}{l}\text { Number of storage } \\
\text { positions }(42,000) \text { and } 1 \\
\text { demand rate (450 } \\
\text { transactions/hour) }\end{array}$ & 32 & $\begin{array}{c}\text { Palletised } \\
\text { unit load }\end{array}$ \\
\hline $\begin{array}{l}\text { Ekren } \text { et al. } \\
\quad(2010)\end{array}$ & B & "tier to tier" & $\begin{array}{c}\text { Simulation } \\
\text { model using } \\
\text { Arena software }\end{array}$ & $\begin{array}{l}\text { Number of storage } \\
\text { positions }(47,628)\end{array}$ & 160 & $\begin{array}{c}\text { Palletised } \\
\text { unit load }\end{array}$ \\
\hline $\begin{array}{l}\text { Ekren } \\
(2011)\end{array}$ & B & "tier to tier" & $\begin{array}{c}\text { Simulation } \\
\text { model using } \\
\text { Arena software }\end{array}$ & $\begin{array}{l}\text { Number of storage } \\
\text { positions }(47,628)\end{array}$ & 55 & $\begin{array}{l}\text { Palletised } \\
\text { unit load }\end{array}$ \\
\hline $\begin{array}{l}\text { Ekren and } \\
\text { Heragu } \\
(2011)\end{array}$ & A & "tier to tier" & $\begin{array}{c}\text { Simulation } \\
\text { model using } \\
\text { Arena software }\end{array}$ & $\begin{array}{c}\text { Number of storage } \\
\text { positions }(47,628) \text { and } 2 \\
\text { demand rates }(450 ; 500 \\
\text { transactions/hour) }\end{array}$ & 110 & $\begin{array}{l}\text { Palletised } \\
\text { unit load }\end{array}$ \\
\hline $\begin{array}{l}\text { Heragu et } \\
\text { al. }(2011)\end{array}$ & $\mathrm{B}$ & "tier captive" & $\begin{array}{l}\text { Analytical } \\
\text { model validated } \\
\text { through } \\
\text { simulation }\end{array}$ & $\begin{array}{l}\text { Number of storage } \\
\text { positions }(20,000) \text { and } 1 \\
\text { demand rate }(500 ; \\
\text { transactions/hour) }\end{array}$ & 4 & $\begin{array}{c}\text { Palletised } \\
\text { unit load }\end{array}$ \\
\hline
\end{tabular}

A: papers that compare AVS/RS with AS/RS

$\mathrm{B}$ : papers that analyse system performance according to different rack configurations

Table 2. Rack configuration data.

\begin{tabular}{ccccccccc}
\hline $\begin{array}{c}\text { Rack } \\
\text { configuration } \\
(R C)\end{array}$ & $\begin{array}{c}\text { Number } \\
\text { of } \\
\text { tiers }(T)\end{array}$ & $\begin{array}{c}\text { Number } \\
\text { of aisles } \\
(A)\end{array}$ & $\begin{array}{c}\text { Number of } \\
\text { columns } \\
(C)\end{array}$ & $\begin{array}{c}\text { ETH }_{V} \\
\text { [retrievals/ } \\
\text { hour*aisle] }\end{array}$ & $\begin{array}{c}\text { ETH }_{L} \\
\text { [retrievals/ } \\
\text { hour*aisle] }\end{array}$ & $\begin{array}{c}\text { Expected } \\
\text { bottleneck }\end{array}$ & $\begin{array}{c}\text { ETH }_{A} \\
\text { [retrievals/ } \\
\text { hour*aisle] }\end{array}$ & $\begin{array}{c}\text { ETH } \\
\text { [retrievals/ } \\
\text { hour] }\end{array}$ \\
\hline 1 & 8 & 4 & 157 & 459 & 901 & Vehicles & 459 & 1836 \\
2 & 8 & 8 & 79 & 785 & 901 & Vehicles & 785 & 6280 \\
3 & 8 & 16 & 40 & 1217 & 901 & Lift & 901 & 14,416 \\
4 & 10 & 4 & 125 & 692 & 829 & Vehicles & 692 & 2768 \\
5 & 10 & 8 & 63 & 1149 & 829 & Lift & 829 & 6632 \\
6 & 10 & 16 & 32 & 1714 & 829 & Lift & 829 & 13,264 \\
7 & 12 & 4 & 105 & 953 & 769 & Lift & 769 & 3076 \\
8 & 12 & 8 & 53 & 1543 & 769 & Lift & 769 & 6152 \\
9 & 12 & 16 & 27 & 2234 & 769 & Lift & 769 & 12,304 \\
\hline
\end{tabular}


Table 3. Data used in simulation experiments.

\begin{tabular}{ccc}
\hline Variable & Unit of measure & Data \\
\hline$\mu_{w}$ & $\mathrm{~m}$ & 0.5 \\
$\mu_{h}$ & $\mathrm{~m}$ & 0.8 \\
$\nu_{V}$ & $\mathrm{~m} / \mathrm{s}$ & 1.5 \\
$\nu_{L}$ & $\mathrm{~m} / \mathrm{s}$ & 5 \\
$\gamma_{V}$ & $\mathrm{~s}$ & 7 \\
$\gamma_{L}$ & $\mathrm{~s}$ & 1 \\
$\varepsilon_{V}$ & $\mathrm{~s}$ & 3 \\
$\varepsilon_{L}$ & $\mathrm{~s}$ & 2 \\
$H_{\max }$ & $\mathrm{m}$ & 10 \\
$L_{\max }$ & $\mathrm{m}$ & 80 \\
\hline
\end{tabular}

Table 4. Simulation results for a single aisle.

\begin{tabular}{|c|c|c|c|c|c|c|c|c|c|c|}
\hline$E x$. & $R C$ & $\begin{array}{c}\lambda_{A} \\
\text { [retrievals/ } \\
\text { hours*aisle] }\end{array}$ & $\begin{array}{c}\text { ETH }_{A} \\
\text { [retrievals/ } \\
\text { hours*aisle] }\end{array}$ & $\begin{array}{c}\text { Throughput } \\
\text { performance } \\
\text { [retrievals } \\
\text { /hours*aisle] }\end{array}$ & $\begin{array}{c}\text { Average } \\
\text { waiting time } \\
\text { for the } \\
\text { vehicle }[\mathrm{s}]\end{array}$ & $\begin{array}{c}\text { Average } \\
\text { waiting time } \\
\text { for the lift } \\
{[s]}\end{array}$ & $\begin{array}{c}\text { Average } \\
\text { cycle time } \\
{[s]}\end{array}$ & $\begin{array}{c}\text { Average } \\
\text { flow time } \\
{[s]}\end{array}$ & $\begin{array}{c}\text { Vehicle } \\
\text { utilisation }\end{array}$ & $\begin{array}{c}\text { Lift } \\
\text { utilisation }\end{array}$ \\
\hline 1 & 1 & 100 & 459 & 100 & 9.91 & 0.23 & 66.92 & 76.83 & 0.22 & 0.11 \\
\hline 2 & 2 & 100 & 785 & 100 & 3.00 & 0.23 & 40.41 & 43.41 & 0.13 & 0.11 \\
\hline 3 & 3 & 100 & 901 & 100 & 1.16 & 0.22 & 27.90 & 29.06 & 0.08 & 0.11 \\
\hline 4 & 4 & 100 & 692 & 100 & 5.00 & 0.28 & 56.65 & 61.65 & 0.14 & 0.12 \\
\hline 5 & 5 & 100 & 829 & 100 & 1.67 & 0.28 & 35.97 & 37.64 & 0.09 & 0.12 \\
\hline 6 & 6 & 100 & 829 & 100 & 0.72 & 0.27 & 25.63 & 26.35 & 0.06 & 0.12 \\
\hline 7 & 7 & 100 & 769 & 100 & 2.98 & 0.33 & 50.36 & 53.34 & 0.11 & 0.13 \\
\hline 8 & 8 & 100 & 769 & 100 & 1.08 & 0.33 & 33.03 & 34.11 & 0.07 & 0.13 \\
\hline 9 & 9 & 100 & 769 & 100 & 0.49 & 0.34 & 24.37 & 24.86 & 0.04 & 0.13 \\
\hline 10 & 1 & 250 & 459 & 250 & 45.50 & 0.64 & 67.29 & 112.80 & 0.54 & 0.28 \\
\hline 11 & 2 & 250 & 785 & 250 & 9.96 & 0.65 & 41.32 & 51.28 & 0.31 & 0.28 \\
\hline 12 & 3 & 250 & 901 & 250 & 3.36 & 0.65 & 28.33 & 31.69 & 0.20 & 0.28 \\
\hline 13 & 4 & 250 & 692 & 250 & 17.78 & 0.82 & 57.17 & 74.95 & 0.36 & 0.30 \\
\hline 14 & 5 & 250 & 829 & 250 & 5.00 & 0.82 & 36.52 & 41.52 & 0.22 & 0.30 \\
\hline 15 & 6 & 250 & 829 & 250 & 1.47 & 0.83 & 23.86 & 25.33 & 0.13 & 0.30 \\
\hline 16 & 7 & 250 & 769 & 250 & 9.54 & 1.03 & 51.02 & 60.56 & 0.26 & 0.32 \\
\hline 17 & 8 & 250 & 769 & 250 & 3.03 & 1.03 & 33.71 & 36.74 & 0.16 & 0.32 \\
\hline 18 & 9 & 250 & 769 & 250 & 1.28 & 1.04 & 25.05 & 26.33 & 0.11 & 0.32 \\
\hline 19 & 1 & 500 & 459 & 459 & 2954 & 1.44 & 68.17 & 3022 & 1 & 0.51 \\
\hline 20 & 2 & 500 & 785 & 500 & 34.09 & 1.66 & 42.36 & 76.45 & 0.64 & 0.55 \\
\hline 21 & 3 & 500 & 901 & 500 & 8.57 & 1.76 & 29.45 & 38.02 & 0.41 & 0.55 \\
\hline 22 & 4 & 500 & 692 & 500 & 74.61 & 2.26 & 58.66 & 133.27 & 0.72 & 0.60 \\
\hline 23 & 5 & 500 & 829 & 500 & 12.94 & 2.39 & 38.11 & 51.05 & 0.44 & 0.60 \\
\hline 24 & 6 & 500 & 829 & 500 & 4.51 & 2.64 & 28.02 & 32.53 & 0.29 & 0.60 \\
\hline 25 & 7 & 500 & 769 & 500 & 27.38 & 3.14 & 53.18 & 80.56 & 0.52 & 0.65 \\
\hline 26 & 8 & 500 & 769 & 500 & 7.10 & 3.56 & 36.27 & 43.37 & 0.32 & 0.65 \\
\hline 27 & 9 & 500 & 769 & 500 & 2.86 & 3.89 & 27.91 & 30.77 & 0.22 & 0.65 \\
\hline 28 & 1 & 750 & 459 & 459 & 35,264 & 1.49 & 68.11 & 35,445 & 1 & 0.51 \\
\hline 29 & 2 & 750 & 785 & 750 & 389.41 & 4.17 & 44.83 & 434.27 & 0.95 & 0.83 \\
\hline 30 & 3 & 750 & 901 & 750 & 20.67 & 6.18 & 33.86 & 54.53 & 0.61 & 0.83 \\
\hline 31 & 4 & 750 & 692 & 692 & 7398 & 5.29 & 61.64 & 7460 & 1 & 0.83 \\
\hline 32 & 5 & 750 & 829 & 750 & 33.82 & 15.32 & 51.01 & 84.83 & 0.65 & 0.91 \\
\hline 33 & 6 & 750 & 829 & 750 & 6.34 & 19.52 & 42.54 & 48.86 & 0.39 & 0.91 \\
\hline 34 & 7 & 750 & 769 & 750 & 99.50 & 67.36 & 117.35 & 216.85 & 0.78 & 0.97 \\
\hline 35 & 8 & 750 & 769 & 750 & 14.89 & 81.71 & 114.39 & 129.28 & 0.48 & 0.97 \\
\hline 36 & 9 & 750 & 769 & 750 & 5.21 & 83.96 & 107.97 & 113.18 & 0.33 & 0.97 \\
\hline 37 & 1 & 1000 & 459 & 459 & 17,534 & 1.44 & 68.16 & 17,602 & 1 & 0.51 \\
\hline 38 & 2 & 1000 & 785 & 785 & 7033 & 4.10 & 44.79 & 7078 & 1 & 0.87 \\
\hline 39 & 3 & 1000 & 901 & 901 & 57.58 & 3192 & 3219 & 3277 & 0.82 & 1 \\
\hline 40 & 4 & 1000 & 692 & 692 & 10,030 & 4.48 & 60.87 & 10,091 & 1 & 0.83 \\
\hline 41 & 5 & 1000 & 829 & 829 & 115.11 & 5453 & 5488 & 5603 & 0.87 & 1 \\
\hline 42 & 6 & 1000 & 829 & 829 & 15.49 & 5554 & 5578 & 5593 & 0.58 & 1 \\
\hline 43 & 7 & 1000 & 769 & 769 & 1939 & 5861 & 5910 & 7849 & 1 & 1 \\
\hline 44 & 8 & 1000 & 769 & 769 & 27.85 & 7494 & 7526 & 7554 & 0.65 & 1 \\
\hline 45 & 9 & 1000 & 769 & 769 & 8.10 & 7520 & 7542 & 7550 & 0.45 & 1 \\
\hline
\end{tabular}


Table 5. Results for the 64 rack configurations considered in the application of the design framework to a real case. The alternatives explored by the framework are highlighted in grey.

\begin{tabular}{|c|c|c|c|c|c|c|c|}
\hline $\begin{array}{c}\text { Rack } \\
\text { configuration } \\
(R C)\end{array}$ & $\begin{array}{c}\text { Number of } \\
\text { aisles }(A)\end{array}$ & $\begin{array}{c}\text { Number of } \\
\text { tiers }(T)\end{array}$ & $\begin{array}{l}\text { Number of } \\
\text { columns }(C)\end{array}$ & $\begin{array}{c}\text { Number of } \\
\text { storage } \\
\text { positions }(n)\end{array}$ & $\begin{array}{c}\text { ETH }_{S} \\
\text { [retrievals/ } \\
\text { hour] }\end{array}$ & $\begin{array}{c}\text { Average flow } \\
\text { time }[s]\end{array}$ & $\begin{array}{c}\text { Annualised } \\
\text { cost }[€]\end{array}$ \\
\hline 1 & 4 & 8 & 149 & 9536 & n.a. & n.a. & n.a. \\
\hline 2 & 4 & 9 & 132 & 9504 & n.a. & n.a. & n.a. \\
\hline 3 & 4 & 10 & 119 & 9520 & n.a. & n.a. & n.a. \\
\hline 4 & 4 & 11 & 108 & 9504 & n.a. & n.a. & n.a. \\
\hline 5 & 4 & 12 & 99 & 9504 & n.a. & n.a. & n.a. \\
\hline 6 & 4 & 13 & 92 & 9568 & n.a. & n.a. & n.a. \\
\hline 7 & 4 & 14 & 85 & 9520 & n.a. & n.a. & n.a. \\
\hline 8 & 4 & 15 & 80 & 9600 & n.a. & n.a. & n.a. \\
\hline 9 & 5 & 8 & 119 & 9520 & n.a. & n.a. & n.a. \\
\hline 10 & 5 & 9 & 106 & 9540 & n.a. & n.a. & n.a. \\
\hline 11 & 5 & 10 & 95 & 9500 & n.a. & n.a. & n.a. \\
\hline 12 & 5 & 11 & 87 & 9570 & n.a. & n.a. & n.a. \\
\hline 13 & 5 & 12 & 80 & 9600 & n.a. & n.a. & n.a. \\
\hline 14 & 5 & 13 & 74 & 9620 & n.a. & n.a. & n.a. \\
\hline 15 & 5 & 14 & 68 & 9520 & n.a. & n.a. & n.a. \\
\hline 16 & 5 & 15 & 64 & 9600 & 3678 & 7050 & 230,040 \\
\hline 17 & 6 & 8 & 99 & 9504 & n.a. & n.a. & n.a. \\
\hline 18 & 6 & 9 & 88 & 9504 & n.a. & n.a. & n.a. \\
\hline 19 & 6 & 10 & 80 & 9600 & n.a. & n.a. & n.a. \\
\hline 20 & 6 & 11 & 72 & 9504 & n.a. & n.a. & n.a. \\
\hline 21 & 6 & 12 & 66 & 9504 & 4847 & 76.88 & 237,595 \\
\hline 22 & 6 & 13 & 61 & 9516 & 4693 & 69.71 & 245,708 \\
\hline 23 & 6 & 14 & 57 & 9576 & 4549 & 67.53 & 254,398 \\
\hline 24 & 6 & 15 & 53 & 9540 & 4414 & 69.42 & 262,619 \\
\hline 25 & 7 & 8 & 85 & 9520 & n.a. & n.a. & n.a. \\
\hline 26 & 7 & 9 & 76 & 9576 & n.a. & n.a. & n.a. \\
\hline 27 & 7 & 10 & 68 & 9520 & n.a. & n.a. & n.a. \\
\hline 28 & 7 & 11 & 62 & 9548 & 5848 & 63.64 & 256,687 \\
\hline 29 & 7 & 12 & 57 & 9576 & 5654 & 56.77 & 266,221 \\
\hline 30 & 7 & 13 & 53 & 9646 & 5475 & 52.58 & 276,359 \\
\hline 31 & 7 & 14 & 49 & 9604 & 5307 & 49.72 & 285,950 \\
\hline 32 & 7 & 15 & 46 & 9660 & 5150 & 48.28 & 296,419 \\
\hline 33 & 8 & 8 & 75 & 9600 & n.a. & n.a. & n.a. \\
\hline 34 & 8 & 9 & 66 & 9504 & 7043 & 70.48 & 262,268 \\
\hline 35 & 8 & 10 & 60 & 9600 & 6923 & 58.81 & 273,020 \\
\hline 36 & 8 & 11 & 54 & 9504 & 6684 & 51.09 & 282,835 \\
\hline 37 & 8 & 12 & 50 & 9600 & 6462 & 47.16 & 294,499 \\
\hline 38 & 8 & 13 & 46 & 9568 & 6257 & 44.05 & 305,538 \\
\hline 39 & 8 & 14 & 43 & 9632 & 6065 & 42.15 & 317,502 \\
\hline 40 & 8 & 15 & 40 & 9600 & 5886 & 40.68 & 328,998 \\
\hline 41 & 9 & 8 & 66 & 9504 & 7043 & 69.88 & 274,604 \\
\hline 42 & 9 & 9 & 59 & 9558 & 8082 & 56.45 & 285,923 \\
\hline 43 & 9 & 10 & 53 & 9540 & 7788 & 48.99 & 297,405 \\
\hline 44 & 9 & 11 & 48 & 9504 & 7519 & 44.17 & 309,311 \\
\hline 45 & 9 & 12 & 44 & 9504 & 7280 & 41.06 & 321,906 \\
\hline 46 & 9 & 13 & 41 & 9594 & 7039 & 39.12 & 335,453 \\
\hline 47 & 9 & 14 & 38 & 9576 & 6823 & 37.42 & 348,473 \\
\hline 48 & 9 & 15 & 36 & 9720 & 6621 & 36.51 & 362,797 \\
\hline 49 & 10 & 8 & 60 & 9600 & 8372 & 57.34 & 297,008 \\
\hline 50 & 10 & 9 & 53 & 9540 & 8980 & 48.32 & 309,000 \\
\hline 51 & 10 & 10 & 48 & 9600 & 8654 & 43.43 & 322,717 \\
\hline 52 & 10 & 11 & 44 & 9680 & 8354 & 40.12 & 337,102 \\
\hline 53 & 10 & 12 & 40 & 9600 & 8078 & 37.48 & 350,706 \\
\hline 54 & 10 & 13 & 37 & 9620 & 7821 & 35.74 & 365,368 \\
\hline 55 & 10 & 14 & 34 & 9520 & 7581 & 34.22 & 379,444 \\
\hline 56 & 10 & 15 & 32 & 9600 & 7357 & 33.37 & 394,969 \\
\hline 57 & 11 & 8 & 54 & 9504 & 9900 & 49.12 & 317,791 \\
\hline 58 & 11 & 9 & 48 & 9504 & 9878 & 43.00 & 331,931 \\
\hline 59 & 11 & 10 & 44 & 9680 & 9519 & 39.62 & 348,184 \\
\hline 60 & 11 & 11 & 40 & 9680 & 9190 & 36.81 & 363,578 \\
\hline 61 & 11 & 12 & 36 & 9504 & 8886 & 34.45 & 378,113 \\
\hline 62 & 11 & 13 & 34 & 9724 & 8603 & 33.40 & 395,835 \\
\hline 63 & 11 & 14 & 31 & 9548 & 8339 & 31.99 & 410,997 \\
\hline 64 & 11 & 15 & 29 & 9570 & 8093 & 31.13 & 427,752 \\
\hline
\end{tabular}

Legend: n.a.: the rack configuration does not satisfy the physical constraint 
Table 6. Unit costs.

\begin{tabular}{ccc}
\hline Cost item & Unit of measure & Value \\
\hline Vehicle & $€ /$ unit & 10,000 \\
Lift & $€ /$ unit & 50,000 \\
Storage position & $€ /$ unit & 30 \\
Area & $€ / \mathrm{m}^{2 *}$ year & 50 \\
\hline
\end{tabular}

Table 7. Data for the scenarios examined in the framework application.

\begin{tabular}{cccc}
\hline Scenario & $\begin{array}{c}\text { Maximum number of } \\
\text { storage columns }\left(C_{\max }\right)\end{array}$ & $\begin{array}{c}\lambda \text { [retrievals/ } \\
\text { hours] }\end{array}$ & $\begin{array}{c}\text { TH }_{\text {target }} \\
\text { [retrievals/hours] }\end{array}$ \\
\hline 1 & 55 & 3,000 & 3334 \\
2 & 55 & 4,000 & 4445 \\
3 & 55 & 5,000 & 5556 \\
4 & 75 & 3,000 & 3334 \\
5 & 75 & 4,000 & 4445 \\
6 & 75 & 5,000 & 5556 \\
7 & 95 & 3,000 & 3334 \\
8 & 95 & 4,000 & 4445 \\
9 & 95 & 5,000 & 5556 \\
\hline
\end{tabular}


Table 8. Results of the design framework application for scenarios 1,2 and 3 (i.e. $C_{\max }$ $=55$ ). For each scenario the sequence of rack configurations evaluated is highlighted.

\begin{tabular}{|c|c|c|c|c|c|c|c|c|c|c|c|c|}
\hline \multirow[t]{2}{*}{$R C$} & \multirow[t]{2}{*}{$A$} & \multirow[t]{2}{*}{$T$} & \multirow[t]{2}{*}{$C$} & \multirow[t]{2}{*}{$n$} & \multirow{2}{*}{$\begin{array}{c}E T H_{S} \\
\text { [retrievals/hour] }\end{array}$} & \multicolumn{3}{|c|}{ Average flow time $[\mathrm{s}]$} & \multirow{2}{*}{$\begin{array}{c}\text { Annualised } \\
\text { cost }[€]\end{array}$} & \multicolumn{3}{|c|}{$\begin{array}{c}\text { Rack configurations } \\
\text { evaluated }\end{array}$} \\
\hline & & & & & & SC1 & $S C 2$ & $S C 3$ & & $S C 1$ & $S C 2$ & $S C 3$ \\
\hline 1 & 4 & 8 & 149 & 9536 & n.a. & n.a. & n.a. & n.a. & n.a. & & & \\
\hline 2 & 4 & 9 & 132 & 9504 & n.a. & n.a. & n.a. & n.a. & n.a. & & & \\
\hline 3 & 4 & 10 & 119 & 9520 & n.a. & n.a. & n.a. & n.a. & n.a. & & & \\
\hline 4 & 4 & 11 & 108 & 9504 & n.a. & n.a. & n.a. & n.a. & n.a. & & & \\
\hline 5 & 4 & 12 & 99 & 9504 & n.a. & n.a. & n.a. & n.a. & n.a. & & & \\
\hline 6 & 4 & 13 & 92 & 9568 & n.a. & n.a. & n.a. & n.a. & n.a. & & & \\
\hline 7 & 4 & 14 & 85 & 9520 & n.a. & n.a. & n.a. & n.a. & n.a. & & & \\
\hline 8 & 4 & 15 & 80 & 9600 & n.a. & n.a. & n.a. & n.a. & n.a. & & & \\
\hline 9 & 5 & 8 & 119 & 9520 & n.a. & n.a. & n.a. & n.a. & n.a. & & & \\
\hline 10 & 5 & 9 & 106 & 9540 & n.a. & n.a. & n.a. & n.a. & n.a. & & & \\
\hline 11 & 5 & 10 & 95 & 9500 & n.a. & n.a. & n.a. & n.a. & n.a. & & & \\
\hline 12 & 5 & 11 & 87 & 9570 & n.a. & n.a. & n.a. & n.a. & n.a. & & & \\
\hline 13 & 5 & 12 & 80 & 9600 & n.a. & n.a. & n.a. & n.a. & n.a. & & & \\
\hline 14 & 5 & 13 & 74 & 9620 & n.a. & n.a. & n.a. & n.a. & n.a. & & & \\
\hline 15 & 5 & 14 & 68 & 9520 & n.a. & n.a. & n.a. & n.a. & n.a. & & & \\
\hline 16 & 5 & 15 & 64 & 9600 & n.a. & n.a. & n.a. & n.a. & n.a. & & & \\
\hline 17 & 6 & 8 & 99 & 9504 & n.a. & n.a. & n.a. & n.a. & n.a. & & & \\
\hline 18 & 6 & 9 & 88 & 9504 & n.a. & n.a. & n.a. & n.a. & n.a. & & & \\
\hline 19 & 6 & 10 & 80 & 9600 & n.a. & n.a. & n.a. & n.a. & n.a. & & & \\
\hline 20 & 6 & 11 & 72 & 9504 & n.a. & n.a. & n.a. & n.a. & n.a. & & & \\
\hline 21 & 6 & 12 & 66 & 9504 & n.a. & n.a. & n.a. & n.a. & n.a. & & & \\
\hline 22 & 6 & 13 & 61 & 9516 & n.a. & n.a. & n.a. & n.a. & n.a. & & & \\
\hline 23 & 6 & 14 & 57 & 9576 & n.a. & n.a. & n.a. & n.a. & n.a. & & & \\
\hline 24 & 6 & 15 & 53 & 9540 & 4414 & 48.55 & 69.42 & 10,648 & 262,619 & 1 & 1 & 1 \\
\hline 25 & 7 & 8 & 85 & 9520 & n.a. & n.a. & n.a. & n.a. & n.a. & & 1 & 1 \\
\hline 26 & 7 & 9 & 76 & 9576 & n.a. & n.a. & n.a. & n.a. & n.a. & & & \\
\hline 27 & 7 & 10 & 68 & 9520 & n.a. & n.a. & n.a. & n.a. & n.a. & & & \\
\hline 28 & 7 & 11 & 62 & 9548 & n.a. & n.a. & n.a. & n.a. & n.a. & & & \\
\hline 29 & 7 & 12 & 57 & 9576 & n.a. & n.a. & n.a. & n.a. & n.a. & & $\nabla$ & \\
\hline 30 & 7 & 13 & 53 & 9646 & 5475 & 45.95 & 52.58 & 74.15 & 276,359 & & 2 & 2 \\
\hline 31 & 7 & 14 & 49 & 9604 & 5307 & 43.26 & 49.72 & 81.86 & 285,950 & & & 1 \\
\hline 32 & 7 & 15 & 46 & 9660 & 5150 & 48.93 & 48.28 & 119.28 & 296,419 & & & \\
\hline 33 & 8 & 8 & 75 & 9600 & n.a. & n.a. & n.a. & n.a. & n.a. & & & \\
\hline 34 & 8 & 9 & 66 & 9504 & n.a. & n.a. & n.a. & n.a. & n.a. & & & 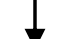 \\
\hline 35 & 8 & 10 & 60 & 9600 & n.a. & n.a. & n.a. & n.a. & n.a. & & & $\nabla$ \\
\hline 36 & 8 & 11 & 54 & 9504 & 6684 & 45.69 & 51.09 & 59.65 & 282,835 & & & 3 \\
\hline 37 & 8 & 12 & 50 & 9600 & 6462 & 42.69 & 47.16 & 54.36 & 294,499 & & & \\
\hline 38 & 8 & 13 & 46 & 9568 & 6257 & 40.11 & 44.05 & 51.28 & 305,538 & & & \\
\hline 39 & 8 & 14 & 43 & 9632 & 6065 & 38.43 & 42.15 & 50.18 & 317,502 & & & \\
\hline 40 & 8 & 15 & 40 & 9600 & 5886 & 36.88 & 40.68 & 50.55 & 328,998 & & & \\
\hline 41 & 9 & 8 & 66 & 9504 & n.a. & n.a. & n.a. & n.a. & n.a. & & & \\
\hline 42 & 9 & 9 & 59 & 9558 & n.a. & n.a. & n.a. & n.a. & n.a. & & & \\
\hline 43 & 9 & 10 & 53 & 9540 & 7788 & 44.32 & 48.99 & 55.63 & 297,405 & & & \\
\hline 44 & 9 & 11 & 48 & 9504 & 7519 & 40.70 & 44.17 & 49.06 & 309,311 & & & \\
\hline 45 & 9 & 12 & 44 & 9504 & 7280 & 38.15 & 41.06 & 45.30 & 321,906 & & & \\
\hline 46 & 9 & 13 & 41 & 9594 & 7039 & 36.47 & 39.12 & 43.23 & 335,453 & & & \\
\hline 47 & 9 & 14 & 38 & 9576 & 6823 & 34.91 & 37.42 & 41.64 & 348,473 & & & \\
\hline 48 & 9 & 15 & 36 & 9720 & 6621 & 34.00 & 36.51 & 41.27 & 362,797 & & & \\
\hline 49 & 10 & 8 & 60 & 9600 & n.a. & n.a. & n.a. & n.a. & n.a. & & & \\
\hline 50 & 10 & 9 & 53 & 9540 & 8980 & 43.87 & 48.32 & 54.45 & 309,000 & & & \\
\hline 51 & 10 & 10 & 48 & 9600 & 8654 & 40.24 & 43.43 & 47.63 & 322,717 & & & \\
\hline 52 & 10 & 11 & 44 & 9680 & 8354 & 37.57 & 40.12 & 43.40 & 337,102 & & & \\
\hline 53 & 10 & 12 & 40 & 9600 & 8078 & 35.34 & 37.48 & 40.33 & 350,706 & & & \\
\hline 54 & 10 & 13 & 37 & 9620 & 7821 & 33.78 & 35.74 & 38.45 & 365,368 & & & \\
\hline 55 & 10 & 14 & 34 & 9520 & 7581 & 32.37 & 34.22 & 39.92 & 379,444 & & & \\
\hline 56 & 10 & 15 & 32 & 9600 & 7357 & 31.53 & 33.37 & 36.27 & 394,969 & & & \\
\hline 57 & 11 & 8 & 54 & 9504 & 9900 & 44.48 & 49.12 & 55.65 & 317,791 & & & \\
\hline 58 & 11 & 9 & 48 & 9504 & 9878 & 39.94 & 43.00 & 47.03 & 331,931 & & & \\
\hline 59 & 11 & 10 & 44 & 9680 & 9519 & 37.25 & 39.62 & 42.54 & 348,184 & & & \\
\hline 60 & 11 & 11 & 40 & 9680 & 9190 & 34.93 & 36.81 & 39.19 & 363,578 & & & \\
\hline 61 & 11 & 12 & 36 & 9504 & 8886 & 32.81 & 34.45 & 36.48 & 378,113 & & & \\
\hline 62 & 11 & 13 & 34 & 9724 & 8603 & 31.88 & 33.40 & 35.40 & 395,835 & & & \\
\hline 63 & 11 & 14 & 31 & 9548 & 8339 & 30.55 & 31.99 & 33.94 & 410,997 & & & \\
\hline 64 & 11 & 15 & 29 & 9570 & 8093 & 29.71 & 31.13 & 33.17 & 427,752 & & & \\
\hline
\end{tabular}

Legend: $n$.a.: the rack configuration does not satisfy the physical constraint 
Table 9. Results of the design framework application for scenarios 4,5 and 6 (i.e. $C_{\max }$ $=75$ ). For each scenario the sequence of rack configurations evaluated is highlighted.

\begin{tabular}{|c|c|c|c|c|c|c|c|c|c|c|c|c|}
\hline \multirow[t]{2}{*}{$R C$} & \multirow[t]{2}{*}{$A$} & \multirow[t]{2}{*}{$T$} & \multirow[t]{2}{*}{$C$} & \multirow[t]{2}{*}{$n$} & \multirow{2}{*}{$\begin{array}{c}\mathrm{ETH}_{S} \\
\text { [retrievals/hour] }\end{array}$} & \multicolumn{3}{|c|}{ Average flow time $[s]$} & \multirow{2}{*}{$\begin{array}{c}\text { Annualised } \\
\text { cost }[€]\end{array}$} & \multicolumn{3}{|c|}{$\begin{array}{c}\text { Rack configurations } \\
\text { evaluated }\end{array}$} \\
\hline & & & & & & SC4 & SC5 & SC6 & & SC4 & $S C 5$ & SC6 \\
\hline 1 & 4 & 8 & 149 & 9536 & n.a. & n.a. & n.a. & n.a. & n.a. & & & \\
\hline 2 & 4 & 9 & 132 & 9504 & n.a. & n.a. & n.a. & n.a. & n.a. & & & \\
\hline 3 & 4 & 10 & 119 & 9520 & n.a. & n.a. & n.a. & n.a. & n.a. & & & \\
\hline 4 & 4 & 11 & 108 & 9504 & n.a. & n.a. & n.a. & n.a. & n.a. & & & \\
\hline 5 & 4 & 12 & 99 & 9504 & n.a. & n.a. & n.a. & n.a. & n.a. & & & \\
\hline 6 & 4 & 13 & 92 & 9568 & n.a. & n.a. & n.a. & n.a. & n.a. & & & \\
\hline 7 & 4 & 14 & 85 & 9520 & n.a. & n.a. & n.a. & n.a. & n.a. & & & \\
\hline 8 & 4 & 15 & 80 & 9600 & n.a. & n.a. & n.a. & n.a. & n.a. & & & \\
\hline 9 & 5 & 8 & 119 & 9520 & n.a. & n.a. & n.a. & n.a. & n.a. & & & \\
\hline 10 & 5 & 9 & 106 & 9540 & n.a. & n.a. & n.a. & n.a. & n.a. & & & \\
\hline 11 & 5 & 10 & 95 & 9500 & n.a. & n.a. & n.a. & n.a. & n.a. & & & \\
\hline 12 & 5 & 11 & 87 & 9570 & n.a. & n.a. & n.a. & n.a. & n.a. & & & \\
\hline 13 & 5 & 12 & 80 & 9600 & n.a. & n.a. & n.a. & n.a. & n.a. & & & \\
\hline 14 & 5 & 13 & 74 & 9620 & 3910 & 74.87 & 1830 & 19,974 & 216,713 & 1 & 1 & 1 \\
\hline 15 & 5 & 14 & 68 & 9520 & 3791 & 67.45 & 4697 & 22,098 & 222,652 & & 1 & 1 \\
\hline 16 & 5 & 15 & 64 & 9600 & 3678 & 63.95 & 7050 & 24,172 & 230,040 & & & \\
\hline 17 & 6 & 8 & 99 & 9504 & n.a. & n.a. & n.a. & n.a. & n.a. & & & \\
\hline 18 & 6 & 9 & 88 & 9504 & n.a. & n.a. & n.a. & n.a. & n.a. & & & \\
\hline 19 & 6 & 10 & 80 & 9600 & n.a. & n.a. & n.a. & n.a. & n.a. & & & \\
\hline 20 & 6 & 11 & 72 & 9504 & 5013 & 68.20 & 93.02 & 499.68 & 229,882 & & 2 & 2 \\
\hline 21 & 6 & 12 & 66 & 9504 & 4847 & 59.96 & 76.88 & 2845 & 237,595 & & & 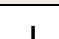 \\
\hline 22 & 6 & 13 & 61 & 9516 & 4693 & 54.64 & 69.71 & 5611 & 245,708 & & & \\
\hline 23 & 6 & 14 & 57 & 9576 & 4549 & 59.19 & 67.53 & 8172 & 254,398 & & & \\
\hline 24 & 6 & 15 & 53 & 9540 & 4414 & 48.55 & 69.42 & 10,648 & 262,619 & & & \\
\hline 25 & 7 & 8 & 85 & 9520 & n.a. & n.a. & n.a. & n.a. & n.a. & & & \\
\hline 26 & 7 & 9 & 76 & 9576 & n.a. & n.a. & n.a. & n.a. & n.a. & & & $\nabla$ \\
\hline 27 & 7 & 10 & 68 & 9520 & 4903 & 61.14 & 76.99 & 111.34 & 247,553 & & & 3 \\
\hline 28 & 7 & 11 & 62 & 9548 & 5848 & 53.74 & 63.64 & 83.22 & 256,687 & & & \\
\hline 29 & 7 & 12 & 57 & 9576 & 5654 & 49.08 & 56.77 & 74.27 & 266,221 & & & \\
\hline 30 & 7 & 13 & 53 & 9646 & 5475 & 45.95 & 52.58 & 74.15 & 276,359 & & & \\
\hline 31 & 7 & 14 & 49 & 9604 & 5307 & 43.26 & 49.72 & 81.86 & 285,950 & & & \\
\hline 32 & 7 & 15 & 46 & 9660 & 5150 & 48.93 & 48.28 & 119.28 & 296,419 & & & \\
\hline 33 & 8 & 8 & 75 & 9600 & 5703 & 71.57 & 101.32 & 210.95 & 253,821 & & & \\
\hline 34 & 8 & 9 & 66 & 9504 & 7043 & 57.80 & 70.48 & 95.32 & 262,268 & & & \\
\hline 35 & 8 & 10 & 60 & 9600 & 6923 & 50.95 & 58.81 & 71.45 & 273,020 & & & \\
\hline 36 & 8 & 11 & 54 & 9504 & 6684 & 45.69 & 51.09 & 59.65 & 282,835 & & & \\
\hline 37 & 8 & 12 & 50 & 9600 & 6462 & 42.69 & 47.16 & 54.36 & 294,499 & & & \\
\hline 38 & 8 & 13 & 46 & 9568 & 6257 & 40.11 & 44.05 & 51.28 & 305,538 & & & \\
\hline 39 & 8 & 14 & 43 & 9632 & 6065 & 38.43 & 42.15 & 50.18 & 317,502 & & & \\
\hline 40 & 8 & 15 & 40 & 9600 & 5886 & 36.88 & 40.68 & 50.55 & 328,998 & & & \\
\hline 41 & 9 & 8 & 66 & 9504 & 7043 & 69.88 & 69.88 & 94.99 & 274,604 & & & \\
\hline 42 & 9 & 9 & 59 & 9558 & 8082 & 56.45 & 56.45 & 67.41 & 285,923 & & & \\
\hline 43 & 9 & 10 & 53 & 9540 & 7788 & 44.32 & 48.99 & 55.63 & 297,405 & & & \\
\hline 44 & 9 & 11 & 48 & 9504 & 7519 & 40.70 & 44.17 & 49.06 & 309,311 & & & \\
\hline 45 & 9 & 12 & 44 & 9504 & 7280 & 38.15 & 41.06 & 45.30 & 321,906 & & & \\
\hline 46 & 9 & 13 & 41 & 9594 & 7039 & 36.47 & 39.12 & 43.23 & 335,453 & & & \\
\hline 47 & 9 & 14 & 38 & 9576 & 6823 & 34.91 & 37.42 & 41.64 & 348,473 & & & \\
\hline 48 & 9 & 15 & 36 & 9720 & 6621 & 34.00 & 36.51 & 41.27 & 362,797 & & & \\
\hline 49 & 10 & 8 & 60 & 9600 & 8372 & 57.34 & 57.34 & 69.05 & 297,008 & & & \\
\hline 50 & 10 & 9 & 53 & 9540 & 8980 & 43.87 & 48.32 & 54.45 & 309,000 & & & \\
\hline 51 & 10 & 10 & 48 & 9600 & 8654 & 40.24 & 43.43 & 47.63 & 322,717 & & & \\
\hline 52 & 10 & 11 & 44 & 9680 & 8354 & 37.57 & 40.12 & 43.40 & 337,102 & & & \\
\hline 53 & 10 & 12 & 40 & 9600 & 8078 & 35.34 & 37.48 & 40.33 & 350,706 & & & \\
\hline 54 & 10 & 13 & 37 & 9620 & 7821 & 33.78 & 35.74 & 38.45 & 365,368 & & & \\
\hline 55 & 10 & 14 & 34 & 9520 & 7581 & 32.37 & 34.22 & 39.92 & 379,444 & & & \\
\hline 56 & 10 & 15 & 32 & 9600 & 7357 & 31.53 & 33.37 & 36.27 & 394,969 & & & \\
\hline 57 & 11 & 8 & 54 & 9504 & 9900 & 44.48 & 49.12 & 55.65 & 317,791 & & & \\
\hline 58 & 11 & 9 & 48 & 9504 & 9878 & 39.94 & 43.00 & 47.03 & 331,931 & & & \\
\hline 59 & 11 & 10 & 44 & 9680 & 9519 & 37.25 & 39.62 & 42.54 & 348,184 & & & \\
\hline 60 & 11 & 11 & 40 & 9680 & 9190 & 34.93 & 36.81 & 39.19 & 363,578 & & & \\
\hline 61 & 11 & 12 & 36 & 9504 & 8886 & 32.81 & 34.45 & 36.48 & 378,113 & & & \\
\hline 62 & 11 & 13 & 34 & 9724 & 8603 & 31.88 & 33.40 & 35.40 & 395,835 & & & \\
\hline 63 & 11 & 14 & 31 & 9548 & 8339 & 30.55 & 31.99 & 33.94 & 410,997 & & & \\
\hline 64 & 11 & 15 & 29 & 9570 & 8093 & 29.71 & 31.13 & 33.17 & 427,752 & & & \\
\hline
\end{tabular}

Legend: $n$.a.: the rack configuration does not satisfy the physical constraint 
Table 10. Results of the design framework application for scenarios 7,8 and 9 (i.e. $C_{\max }$ =95). For each scenario the sequence of rack configurations evaluated is highlighted.

\begin{tabular}{|c|c|c|c|c|c|c|c|c|c|c|c|c|}
\hline \multirow[t]{2}{*}{$R C$} & \multirow[t]{2}{*}{$A$} & \multirow[t]{2}{*}{$T$} & \multirow[t]{2}{*}{$C$} & \multirow[t]{2}{*}{$n$} & \multirow{2}{*}{$\begin{array}{c}\text { ETH }_{S} \\
\text { [retrievals/hour] }\end{array}$} & \multicolumn{3}{|c|}{ Average flow time [s] } & \multirow{2}{*}{$\begin{array}{c}\text { Annualised } \\
\text { cost }[€]\end{array}$} & \multicolumn{3}{|c|}{$\begin{array}{c}\text { Rack configurations } \\
\text { evaluated }\end{array}$} \\
\hline & & & & & & $S C 7$ & $S C 8$ & SC9 & & $S C 7$ & $S C 8$ & SC9 \\
\hline 1 & 4 & 8 & 149 & 9536 & n.a. & n.a. & n.a. & n.a. & n.a. & & & \\
\hline 2 & 4 & 9 & 132 & 9504 & n.a. & n.a. & n.a. & n.a. & n.a. & & & \\
\hline 3 & 4 & 10 & 119 & 9520 & n.a. & n.a. & n.a. & n.a. & n.a. & & & \\
\hline 4 & 4 & 11 & 108 & 9504 & n.a. & n.a. & n.a. & n.a. & n.a. & & & \\
\hline 5 & 4 & 12 & 99 & 9504 & n.a. & n.a. & n.a. & n.a. & n.a. & & & \\
\hline 6 & 4 & 13 & 92 & 9568 & 3128 & 180.85 & 19,963 & 34,313 & 186,614 & 1 & 1 & 1 \\
\hline 7 & 4 & 14 & 85 & 9520 & 3032 & 75,766 & 79,807 & 82,216 & 191,294 & 1 & 1 & 1 \\
\hline 8 & 4 & 15 & 80 & 9600 & 2943 & 1741 & 24,174 & 37693 & 197,054 & & & \\
\hline 9 & 5 & 8 & 119 & 9520 & n.a. & n.a. & n.a. & n.a. & n.a. & & & \\
\hline 10 & 5 & 9 & 106 & 9540 & n.a. & n.a. & n.a. & n.a. & n.a. & $\downarrow$ & $\downarrow$ & $\downarrow$ \\
\hline 11 & 5 & 10 & 95 & 9500 & 3719 & 174.25 & 6649 & 23,471 & 197,701 & 2 & 2 & 2 \\
\hline 12 & 5 & 11 & 87 & 9570 & 4177 & 111.32 & 335.52 & 15,172 & 203,900 & & 3 & 3 \\
\hline 13 & 5 & 12 & 80 & 9600 & 4039 & 87.08 & 317.62 & 17,625 & 210,188 & & 1 & 1 \\
\hline 14 & 5 & 13 & 74 & 9620 & 3910 & 74.87 & 1830 & 19,974 & 216,713 & & & \\
\hline 15 & 5 & 14 & 68 & 9520 & 3791 & 67.45 & 4697 & 22,098 & 222,652 & & & \\
\hline 16 & 5 & 15 & 64 & 9600 & 3678 & 63.95 & 7050 & 24,172 & 230,040 & & & $\downarrow$ \\
\hline 17 & 6 & 8 & 99 & 9504 & n.a. & n.a. & n.a. & n.a. & n.a. & & $\nabla$ & \\
\hline 18 & 6 & 9 & 88 & 9504 & 4263 & 114.96 & 457.20 & 12,860 & 215,825 & & 4 & 4 \\
\hline 19 & 6 & 10 & 80 & 9600 & 5094 & 84.38 & 141.00 & 1000 & 223,322 & & 5 & 5 \\
\hline 20 & 6 & 11 & 72 & 9504 & 5013 & 68.21 & 93.02 & 499.68 & 229,882 & & & 6 \\
\hline 21 & 6 & 12 & 66 & 9504 & 4847 & 59.96 & 76.88 & 2845 & 237,595 & & & 1 \\
\hline 22 & 6 & 13 & 61 & 9516 & 4693 & 54.64 & 69.71 & & 245,708 & & & \\
\hline 23 & 6 & 14 & 57 & 9576 & 4549 & 59.19 & 67.53 & 8172 & 254,398 & & & \\
\hline 24 & 6 & 15 & 53 & 9540 & 4414 & 48.55 & 69.42 & 10,648 & 262,619 & & & $\downarrow$ \\
\hline 25 & 7 & 8 & 85 & 9520 & 4541 & 100.30 & 241.68 & 8533 & 231,551 & & & 7 \\
\hline 26 & 7 & 9 & 76 & 9576 & 5559 & 74.16 & 107.34 & 263.78 & 239,626 & & & 8 \\
\hline 27 & 7 & 10 & 68 & 9520 & 4903 & 61.14 & 76.99 & 111.34 & 247,553 & & & \\
\hline 28 & 7 & 11 & 62 & 9548 & 5848 & 53.74 & 63.64 & 83.22 & 256,687 & & & \\
\hline 29 & 7 & 12 & 57 & 9576 & 5654 & 49.08 & 56.77 & 74.27 & 266,221 & & & \\
\hline 30 & 7 & 13 & 53 & 9646 & 5475 & 45.95 & 52.58 & 74.15 & 276,359 & & & \\
\hline 31 & 7 & 14 & 49 & 9604 & 5307 & 43.26 & 49.72 & 81.86 & 285,950 & & & \\
\hline 32 & 7 & 15 & 46 & 9660 & 5150 & 48.93 & 48.28 & 119.28 & 296,419 & & & \\
\hline 33 & 8 & 8 & 75 & 9600 & 5703 & 71.57 & 101.32 & 210.95 & 253,821 & & & \\
\hline 34 & 8 & 9 & 66 & 9504 & 7043 & 57.80 & 70.48 & 95.32 & 262,268 & & & \\
\hline 35 & 8 & 10 & 60 & 9600 & 6923 & 50.95 & 58.81 & 71.45 & 273,020 & & & \\
\hline 36 & 8 & 11 & 54 & 9504 & 6684 & 45.69 & 51.09 & 59.65 & 282,835 & & & \\
\hline 37 & 8 & 12 & 50 & 9600 & 6462 & 42.69 & 47.16 & 54.36 & 294,499 & & & \\
\hline 38 & 8 & 13 & 46 & 9568 & 6257 & 40.11 & 44.05 & 51.28 & 305,538 & & & \\
\hline 39 & 8 & 14 & 43 & 9632 & 6065 & 38.43 & 42.15 & 50.18 & 317,502 & & & \\
\hline 40 & 8 & 15 & 40 & 9600 & 5886 & 36.88 & 40.68 & 50.55 & 328,998 & & & \\
\hline 41 & 9 & 8 & 66 & 9504 & 7043 & 69.88 & 69.88 & 94.99 & 274,604 & & & \\
\hline 42 & 9 & 9 & 59 & 9558 & 8082 & 56.45 & 56.45 & 67.41 & 285,923 & & & \\
\hline 43 & 9 & 10 & 53 & 9540 & 7788 & 44.32 & 48.99 & 55.63 & 297,405 & & & \\
\hline 44 & 9 & 11 & 48 & 9504 & 7519 & 40.70 & 44.17 & 49.06 & 309,311 & & & \\
\hline 45 & 9 & 12 & 44 & 9504 & 7280 & 38.15 & 41.06 & 45.30 & 321,906 & & & \\
\hline 46 & 9 & 13 & 41 & 9594 & 7039 & 36.47 & 39.12 & 43.23 & 335,453 & & & \\
\hline 47 & 9 & 14 & 38 & 9576 & 6823 & 34.91 & 37.42 & 41.64 & 348,473 & & & \\
\hline 48 & 9 & 15 & 36 & 9720 & 6621 & 34.00 & 36.51 & 41.27 & 362,797 & & & \\
\hline 49 & 10 & 8 & 60 & 9600 & 8372 & 57.34 & 57.34 & 69.05 & 297,008 & & & \\
\hline 50 & 10 & 9 & 53 & 9540 & 8980 & 43.87 & 48.32 & 54.45 & 309,000 & & & \\
\hline 51 & 10 & 10 & 48 & 9600 & 8654 & 40.24 & 43.43 & 47.63 & 322,717 & & & \\
\hline 52 & 10 & 11 & 44 & 9680 & 8354 & 37.57 & 40.12 & 43.40 & 337,102 & & & \\
\hline 53 & 10 & 12 & 40 & 9600 & 8078 & 35.34 & 37.48 & 40.33 & 350,706 & & & \\
\hline 54 & 10 & 13 & 37 & 9620 & 7821 & 33.78 & 35.74 & 38.45 & 365,368 & & & \\
\hline 55 & 10 & 14 & 34 & 9520 & 7581 & 32.37 & 34.22 & 39.92 & 379,444 & & & \\
\hline 56 & 10 & 15 & 32 & 9600 & 7357 & 31.53 & 33.37 & 36.27 & 394,969 & & & \\
\hline 57 & 11 & 8 & 54 & 9504 & 9900 & 44.48 & 49.12 & 55.65 & 317,791 & & & \\
\hline 58 & 11 & 9 & 48 & 9504 & 9878 & 39.94 & 43.00 & 47.03 & 331,931 & & & \\
\hline 59 & 11 & 10 & 44 & 9680 & 9519 & 37.25 & 39.62 & 42.54 & 348,184 & & & \\
\hline 60 & 11 & 11 & 40 & 9680 & 9190 & 34.93 & 36.81 & 39.19 & 363,578 & & & \\
\hline 61 & 11 & 12 & 36 & 9504 & 8886 & 32.81 & 34.45 & 36.48 & 378,113 & & & \\
\hline 62 & 11 & 13 & 34 & 9724 & 8603 & 31.88 & 33.40 & 35.40 & 395,835 & & & \\
\hline 63 & 11 & 14 & 31 & 9548 & 8339 & 30.55 & 31.99 & 33.94 & 410,997 & & & \\
\hline 64 & 11 & 15 & 29 & 9570 & 8093 & 29.71 & 31.13 & 33.17 & 427,752 & & & \\
\hline
\end{tabular}

Legend: $n$.a.: the rack configuration does not satisfy the physical constraint 